\title{
From Microelectronics to Molecular Spintronics: an explorer's traveling guide
}

\author{
J. Ferrer \\ Departamento de Física, Universidad de Oviedo, 33007 Oviedo, Spain \\ V. M. García-Suárez \\ Department of Physics, Lancaster University, Lancaster LA1 4YB, U.K.
}

(Dated: June 21, 2008)

\begin{abstract}
We present in this article a broad overview of the fields of molecular electronics and molecular spintronics. This is supplemented by an pedagogical introduction which presents basic concepts of electronic transport and of microelectronics, which intends to show the most pressing issues that face the microelectronics industry.
\end{abstract}

\section{INTRODUCTION}

The fabulous development of Software Science in the past three decades is possibly the main driver of the changes that our world has undertaken. These developments affect virtually all aspects of society, from social relations to banking, from journalism to publicity, and from engineering to basic science research. We scientist spend many hours in front of the screen of a computer, regardless of whether we are theorists or experimentalists. Many of the wealthiest people in the world have made their fortunes because of the success of their software companies[1].

The work horse behind this tremendous explosion of software programs that inundate our lives is the computer, whose architecture and individual components have experienced a similar transformation rate. Indeed, the pressure for ever more computing power can be seen from the growth in the number of gigantic data centers across the world[2]. The key aspects that express the power of a computer are its ability to process information (logics), and to store/retrieve such information (memory).

The CPU chip is the element of a computer in charge of performing the logical operations. The chip is an extremely complex integrated circuit made of silicon which includes a huge number of transistors and connecting circuitry. The demand for increasing processing power can only be achieved by a concomitant increase in the number of individual elements in a single chip, while maintaining its size. Nowadays, a chip with an area of about $1 \mathrm{~cm}^{2}$ encloses about $10^{9}$ transistors. This is achieved by decreasing the size of these elements. Indeed, the length of the channel that joins the source and the drain in a transistor is used to name the technology generation of a chip. Current leading edge chips use the 45 nanometer technology [3], while the microelectronics industry roadmaps expect to be able to reach the $10 \mathrm{~nm}$ technology limit|4]. Beyond this, the quantum nature of atoms and molecules is expected to determine increasingly the behavior of those components.

Hardware companies fabricate chips in large and costly fabrication plants[5]. Those chips are manufactured with the use of optical lithography technology. This technique stamps the transistors and circuitry of the chip on silicon wafers in a series of steps that lay out the different layers that make up the transistor, and which are jointly called a process flow.

Information is stored using a variety of technologies. Some of them are purely electrical, as is the case for DRAM or flash memories. Spintronics designs the technologies that use the spin degrees of freedom of the electrons. This is the case of the magnetoresistive technology, used to store data in hard disk drives. The aspects that must be considered when choosing one technology or another are storage capacity (which determines the cost), speed to retrieve/store data and energy consumption.

DRAM memories are made of a huge array of capacitors. It is the fastest type of memory, since the access time is of about $5 \mathrm{~ns}$, but can achieve lower data densities. An important drawback is that the capacitors discharge very fast and must be recharged continually. DRAM memory therefore consumes more energy than the rest. It is also volatile, since all data are lost when the memory is disconnected from the power supply. The array in Flash memories is basically made of transistors. This type of memory achieves higher data storage densities than DRAM, but are slower, with access times in the order 100 ns. Flash memory has the important drawback that it degrades with time.

Spintronics technology is based on the magnetoresistive effect. Bits are stored magnetic particles, located on the surface of a rotating disk. These are read or written with magnetic heads that scan the surface of the disk. Magnetoresistive technology can handle quite higher data densities than the rest, and it is therefore the cheapest). It also requires lower energy consumption, but can access data at the slow pace of $1 \mathrm{bit} / \mathrm{ms}$. This is because hard disks rotate at about 15.000 RPM. Magnetic RAM memories (MRAM) use a direct access to the magnetic bits, avoiding the slow rotation/scan procedure. They achieve access times of the order of $10 \mathrm{~ns}$ or even less. MRAM technology is not fully mature yet for its use in commercial devices, but has the promise to bring about the universal memory, which will conjugate huge storage densities, huge speed, and ultra-low energy consumption and cost.

The shrinking size of electronic components in integrated circuits leads to think that the sub-10nm limit will be reached within one decade at most. This limit certainly implies that today's CMOS technology is bound to pass away in the not so long future. On the one hand, lithographic techniques are possibly not the best tool to handle sub-10 $\mathrm{nm}$ devices, since 
the discrete nature of the molecules and atoms will eventually be felt. On the other, the quantum behavior of those devices will possibly force to change completely the architecture paradigms that are used today in the microelectronics, or better to say, the Nanoelectronics industry.

Atomic and molecular physics and chemistry is the realm where quantum effects fully unfold. Since these could only be seen at ultra low temperatures, or under extreme laboratory conditions, quantum physics has been regarded as a basic research field for many years. But the technological developments of the last two decades, brought about mainly by the invention of the Scanning Tunneling Microscope [6], have made it possible to achieve control over quite a few atomic and molecular entities already.

The uselessness of lithography for the fabrication and assembly of sub-10 nm elements leads to ask how to fabricate and assemble them. A possible solution is of course to use the STM to manipulate individual atoms and add them up in a certain manner, which is known as the bottom-up approach. However, this approach is expected to be very difficult and hard to put in practice and it is not even clear wether it will ever become feasible[7]. A more clever alternative involves using molecules as the basic electronic elements and assembling them as if they were a molecular LEGO. This ability defines the field known as molecular electronics, with its promise to open the door to a full new world possibilities.

Even though molecular electronics is a relatively new field the advances on this area of research have been significant. Molecules have a series of advantages over the traditional silicon based lithographic techniques. Besides the obvious huge increase in packing density of electronics components, they can be synthesized easily and with low cost in many cases; they can be grown in three dimensions as opposed to the typical 2D semiconductor lithographic layout; they can self-assemble; they are expected to reduce heat and noise production and, most importantly, they can display a whole new world of properties and behaviors. Molecular assemblies have already been proven to provide many of the archetypical microelectronics functionalities, like diode or transistor behavior or to function as logic gates.

Adding the spin degree of freedom to molecular electronics brings a full new suite of functionalities, in the same way that spintronics complements microelectronics. Furthermore, molecular spintronics may be prove to be useful not only for the fabrication of memory devices, but also for the design of elements for classical or quantum logics. Furthermore, handling only the spin degree of freedom is expected to reduce heat dissipation and therefore to produce ultra-low levels of energy consumption and noise.

While it is not clear yet that Molecular Electronics and Molecular Spintronics will finally prove to be viable technologies, it is quite clear that in case they do, the Quantum physics and chemistry will swap its role from basic to applied science, and physicists and chemists will be work and be regarded as electrical engineers.

We have designed this review article as a general-purpose introduction to molecular electronics and spintronics. We have had in mind a generic audience of chemists, both experimentalists and theoreticians. We have therefore assumed that the readers of this article have not had an intense exposure to the field of electronic transport, but are interested in learning about it and possibly entering the field of molecular electronics and spintronics. We do assume that they have some knowledge on Solid State Physics and, of course, on quantum mechanics and statistical physics at the Graduate level. Theoreticians can read the whole article through. Experimentalists will possibly prefer to skip those few sections where we have dumped the technicalities. They can easily be spotted since they are plagued with formulae, in sharp contrast with the rest, where there is basically none. The article is divided in two big parts. The first is devoted to lay out the basic concepts of electronic transport, of microelectronics, and of spintronics, that are needed to understand the issues at stake. We also comment on the troubles and limitations that afflict the microelectronics industry and which are likely to force the demise of the current CMOS technology. The second part is devoted Molecular Electronics and Spintronics themselves. We first introduce the basic concepts behind molecular electronics, and then explain the most relevant experimental achievements in the field. We finally discuss the methodology behind the most popular theoretical tools used in the field. We finally come to Molecular Spintronics, where we first discuss the devices and architectures that have been realized experimentally, or proposed theoretically, and their possible functionalities. We then present the extensions to Molecular Electronics packages that are needed to simulate spin transport, and end by presenting some of the relevant simulations of molecular spintronics devices that have been performed in the past few years. Extensive monographs on electronic transport in mesoscopic systems can be found elsewhere[8].

\section{BASIC NOTIONS OF ELECTRON TRANSPORT IN A MATERIAL}

\section{Conductance of a bulk material}

To understand the physics behind electron transport, we will discuss in this section a simple model that will bring the essential concepts. The first one consists of a metallic sample of length $L$ and section $A$, whose electronic structure is well described by a single electronic band $\epsilon_{k}$. We assume that the electrons may change from one $k$-state to another because of scattering events with impurities, or with lattice vibrations. The scattering rate is quantified by the relaxation time $\tau_{k}$, which is the average time an electron in state $k$ travels between two scattering events[9]. Imagine now that a DC voltage drop $V$ across the sample is supplied by an external source.

Suppose first that the voltage $V$ is zero. Then the sample is in thermodynamic equilibrium and no net current can be measured. The electrons in the system will fill the states in the 
band following the Fermi distribution function $f(k)$. Imagine now that a finite voltage $V$ is switched on. Then a static and uniform electric field $\vec{E}$ of modulus $E=-V / L$ is felt in the material This field causes that the electronic population of the materials will no longer be in equilibrium, Rather, the states will be filled according to a new distribution function $g_{k}$. The electric field will also cause a net motion of electrons, so that there will be a net current density

$$
\vec{j}=-2 e \int \frac{\mathrm{d} k}{2 \pi} \vec{v}_{k} g_{k}
$$

where $\vec{v}_{k}=\frac{1}{\hbar} \vec{\nabla} \epsilon_{k}$ and $g_{k}$ is the distribution function of electrons. If the material is isotropic, the current density $\vec{j}$ is parallel to the electric field. If in addition, $E$ is small enough, the expression for the current can be simplified to[9]

$$
\begin{aligned}
j=\sigma \mathrm{E} & =2 e^{2} \int \frac{\mathrm{d} k}{2 \pi} \tau_{k} v_{k}^{2}\left(-\frac{\mathrm{d} f_{k}}{\mathrm{~d} \epsilon_{k}}\right) \mathrm{E} \\
& =2 e^{2} \int \mathrm{d} \epsilon N(\epsilon) \tau(\epsilon) v^{2}(\epsilon)\left(-\frac{\mathrm{d} f(\epsilon)}{\mathrm{d} \epsilon}\right) \mathrm{E}
\end{aligned}
$$

where $\sigma$ is the conductivity of the material, $N$ is the density of states and $f_{k}$ is the Fermi distribution function. At low temperatures, the derivative of the Fermi distribution is (minus) a Dirac-delta function that cancels the integral. The conductivity is simply determined by the above magnitudes evaluated at the Fermi energy $\epsilon_{\mathrm{F}}$,

$$
\sigma=2 e^{2} N_{\mathrm{F}} \tau_{\mathrm{F}} v_{\mathrm{F}}^{2}=2 e^{2} N_{\mathrm{F}} v_{\mathrm{F}} l
$$

where the mean free path $l=v_{\mathrm{F}} \tau_{\mathrm{F}}$ is the average length traversed by an electron between two scattering events. The conductance of the sample

$$
G=\frac{A}{L} \sigma=2 e^{2} N_{\mathrm{F}} v_{\mathrm{F}} \frac{l}{L}
$$

depends on the ratio $l / L$. The ballistic regime covers the cases where $l \gg L$ so that the electrons will suffer very few scattering events. In this case the above expression must be replaced by

$$
G=2 e^{2} N_{\mathrm{F}} v_{\mathrm{F}}
$$

On the opposite side, the diffusive regime happens when $l \ll L$ so that each electron that enters the sample on one side will scatter many times before leaving it from the other side. The conductance is in this case small. Notice that the room-temperature mean free path of a metal is of the order of a few nanometers, so that the transport regime of a typical metallic sample is diffusive. In contrast, the room temperature mean free path in a doped semiconductor is much longer, of
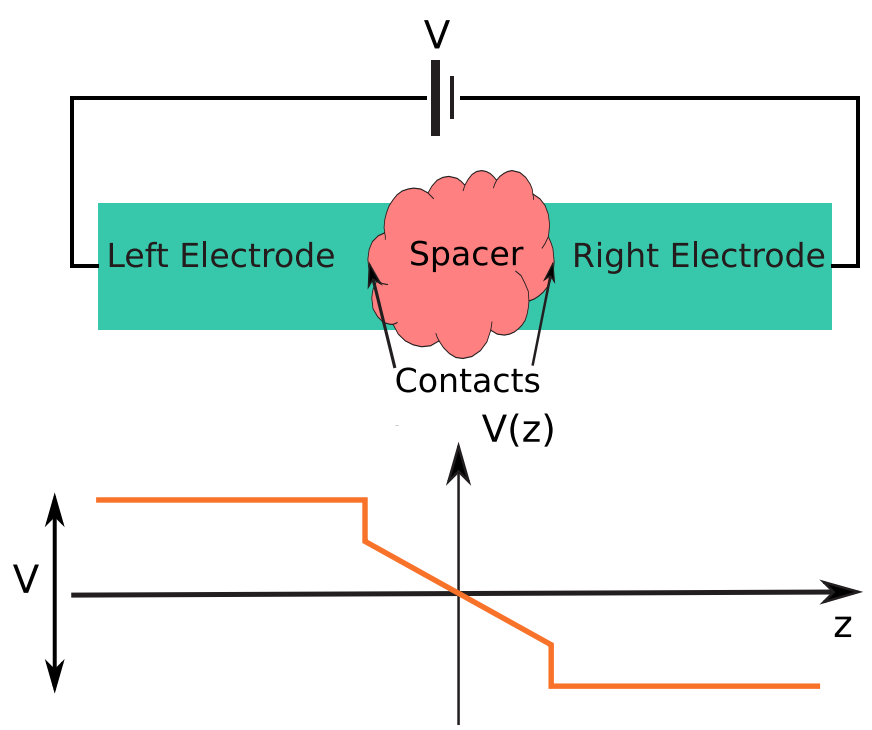

FIG. 1: (Color online) Top panel: schematic plot of a junction, showing the two electrodes, the spacer and the contacts. Bottom panel: voltage drop profile, showing that the contacts and the spacer are the regions of high resistance.

the order of tens of nanometer. The transport regime of some key components in microelectronic chips is currently crossing over from the diffusive to the ballistic regime.

We generalize now the above discussion to a realistic material with a complicated electronic structure, so that there are $N$ bands that cross the Fermi energy. Then the above derivation can be carried through and the conductivity of the material becomes a sum of the contributions of each band

$$
\sigma=2 e^{2} \sum_{n} N_{\mathrm{F} n} \tau_{\mathrm{F} n} v_{\mathrm{F} n}^{2}
$$

\section{Conductance of a one-dimensional ballistic sample: the conduction channel}

We assume now that the section $A$ is so small that the ballistic metallic sample can be regarded as a one-dimensional bar. The criterion to decide if this is so, is that transverse length $d$ is smaller than the Fermi wave length (for a metal, $\lambda_{\mathrm{F}} \simeq 1$ $\mathrm{nm}$; for a doped semiconductor, $\lambda_{\mathrm{F}} \simeq 10-100 \mathrm{~nm}$ ). If this is the case, then the conductance is related to the conductivity through the equation

$$
G=\frac{1}{L} \sigma
$$

Further, the density of states at the Fermi energy can be expressed as $N_{\mathrm{F}}=\frac{1}{h v_{\mathrm{F}}}$ so that the Fermi velocity cancels out and the conductance can be written as

$$
G=N G_{0}
$$



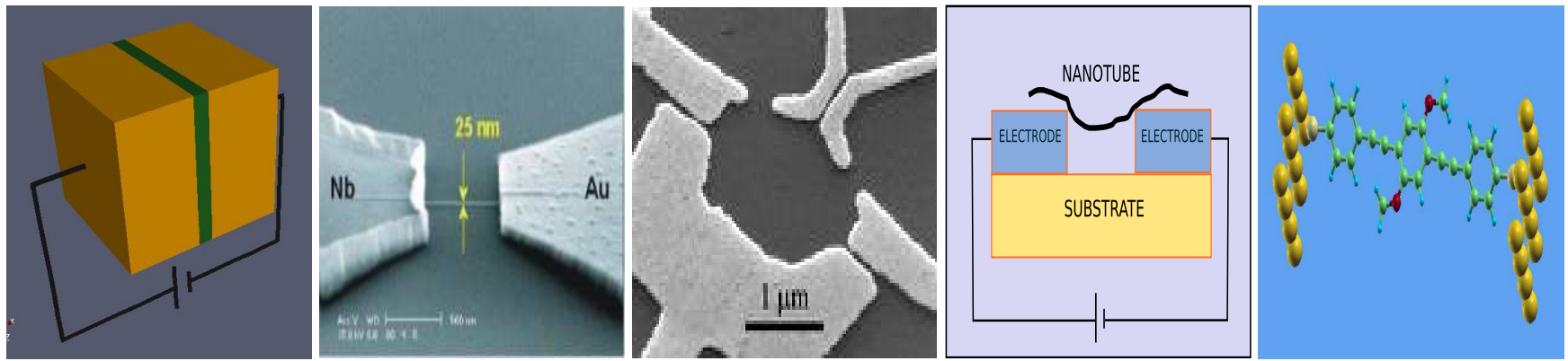

FIG. 2: (Color online) Junctions that are characterized by different spacers. From left to right: a planar junction; a metallic bar; a quantum dot; a carbon nanotube; an organic molecule.

where $G_{0}=2 e^{2} / h$ is defined as the conductance quantum unit. This universal formula states that the normalized conductance is equal to the number of bands crossing the Fermi energy. These can be viewed as tracks that the electrons can use to move through the sample and are therefore called conduction channels.

Imagine finally that the ballistic bar is connected to two identical electrodes, that supply the voltage bias $V$ to the bar, and are the source and drain of electrons. There will clearly be a mismatch between the electronic eigenstates of the electrodes and the bar that will be felt by the electrons when they cross the contacts. This mismatch at both sides is a source of scattering for the electrons that cross the contacts, which can be quantified by the transmission probability $T_{n}$ that an incoming electron will scatter to channel $n$ at the bar, or that an outgoing electron at channel $n$ will leave the bar[10]. Then

$$
G=G_{0} \sum_{n} T_{n}
$$

which is a generalization of the previous formula.

\section{The concept of junction}

The above example of a ballistic bar that bridges two bulk materials is one of the easiest examples of one of the central concepts in this review: that of a junction. A junction is a solid state device which consists of a two metallic bulk materials, called electrodes, that sandwich something called the spacer, as we depict schematically in Fig. 1. The spacer can be anything that permits some electron flow between the electrodes: another material, vacuum, a molecule, a quantum dot, etc. The small regions in space where the spacer and the electrodes attach to each other are called the contacts. The electrodes are subjected to a voltage bias $V$ supplied by an external power, and it is usually assumed that the whole voltage drop occurs at the region in space that comprises the contacts and the spacer, which is therefore called the scattering region.

Transport in the spacer can be ballistic or diffusive, depending on whether its length is larger or shorter than the mean free path. In the first case, the voltage drop occurs entirely at the contacts, while in the second, part of this voltage drop also occurs within the spacer. Correspondingly, the resistance in the first case is entirely due to the contacts (this is called contact resistance), while in the second it is due to both contacts and scattering events within the spacer.

A junction can have any geometry, but it is usually assumed that the electrodes are very large (infinite as a matter of fact). Planar junctions are those where the transverse section of the spacer and the contacts is much larger than $\lambda_{F}^{2}$. On the opposite limit, a point contact is a junction whose section is of the order of $\lambda_{F}^{2}$ or even smaller. An example is the ballistic bar discussed in the previous section, as well as molecular junctions or quantum dots.

Atomic or molecular junctions represent the extreme limit of point contacts, where the spacer consists of a single atom or molecule. Electronic transport through a single atomic contact was studied numerically by Lang who found that $G \sim G_{0}[12]$. Later on, Ferrer[13, 14] demonstrated that, under specific circumstances, the conductance of a single atom junction could indeed be quantized

$$
G=n G_{0}
$$

with $n$ being the number of channels. This result was later generalized by Cuevas et al[15], who defined properly the concept of conducting channel at the contact and accounted for their finite transmission,

$$
G=G_{0} \sum_{n} T_{n}
$$

Molecular junctions were introduced by Aviram and Ratner[16], who proposed their use as current rectifiers.

\section{Electronic states in a molecular junction or a quantum dot}

Molecular junctions and quantum dots have a similar electronic spectra and potential landscape. We sketch an example in Fig. 3(a), where the two electrodes are subjected to a voltage bias $V$ that shifts their Fermi energy levels by $\pm e V / 2$. 

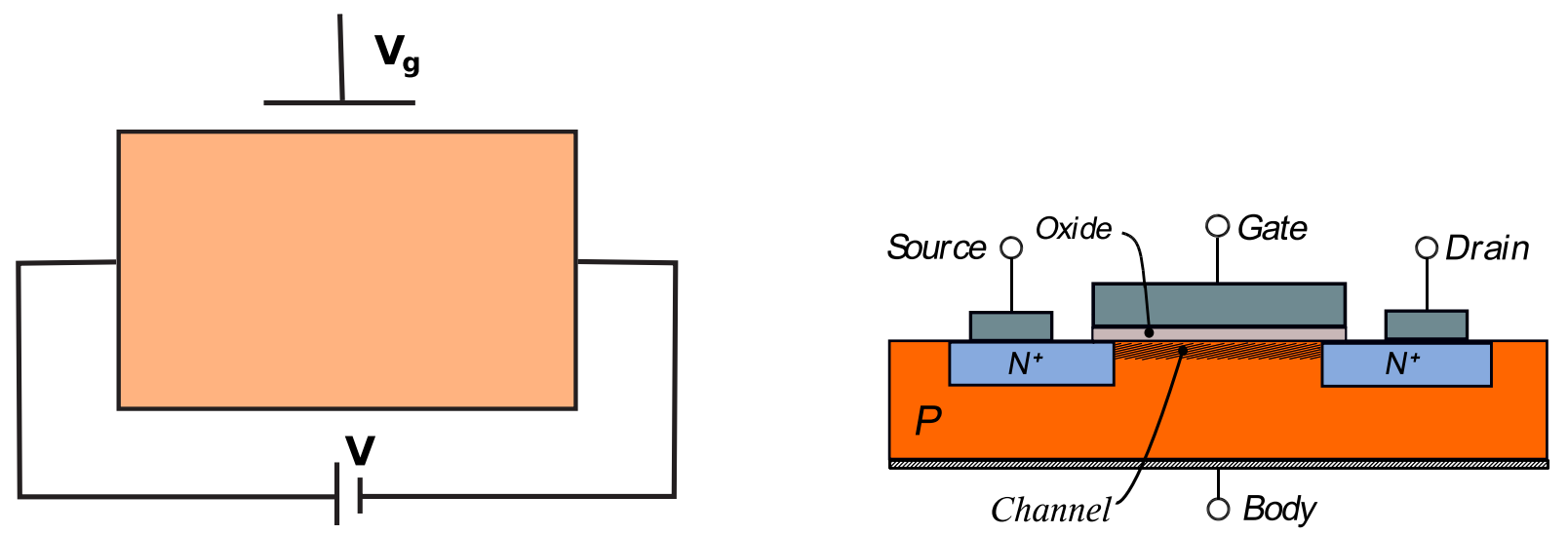

FIG. 3: (Color online) Schematic drawings of several microelectronics components (a) A metallic device with resistance R, attached to a power supply and influenced by a gate. (b) A Field Effect transistor.

As shown in the figure, these junctions share the fact that the eigenstates at the spacer have a discrete energy spectra, which is separated from the continuum of energy states at the electrodes by energy barriers at the contacts. The population of electrons in the electrodes can be described by Fermi distribution functions, whereby all states up to the respective Fermi energy are filled. Because of the Fermi exclusion principle, only those electrons from the left electrode that have an energy in the window $(-e V / 2,+e V / 2))$ can hop into the spacer and eventually to the right electrode.

There is no way to define a Fermi energy at the spacer since the spacer is in contact with two electron reservoirs at different chemical potentials and therefore the spacer is a system out of equilibrium whose distribution function is not the Fermi function. In the case of the figure, there is a single state at the spacer in the energy window $-e V / 2,+e V / 2)$, which is separated from the pristine $E_{F}$ by the energy $E_{C}$. Electrons coming from the left electrode must hop into this state and then move on to the right electrode.

Up to now, we have assumed that the energy levels at the spacer are discrete, but this is not exactly true: the coupling to the electrodes furnishes them with a finite linewidth $\Gamma$, see Fig. 3(b), which accounts for the fact that any electron at the spacer has a finite probability of leaving it, or the other way round. Indeed, the residence time of an electron at the spacer can be estimated from $\Delta t_{R} \Gamma \simeq \hbar$, where $\Gamma$ is the linewidth of the resonance or, alternatively, the probability per unit time that an electron hop into or outside the spacer.

The transparent regime corresponds to those cases where the energy barriers are low enough that the electrons hop in and out of the spacer easily and frequently, so that the discrete energy levels become wide resonances, $\Gamma \sim$. The tunneling regime corresponds to the opposite case, where the barriers are quite high, and electrons can only reach the spacer via tunneling events. In this case, the discrete energy levels as sharp resonances, e. g.: their linewidth $\Gamma$ is . Incoming electrons from one electrode will have a chance to pass to the other only if their energy equals the energy of one of the resonances, an effect which is called resonant tunneling. In other words, residence times are short in the transparent regime and long in the tunneling regime.

When the residence time in a molecular junction or quantum dot is long, two additional complications may occurs. First, electrons may suffer scattering events with an atomic vibration or with another electron, changing its energy. In this case, the phase of wave function changes and quantum coherence effects are lost or at least blurred. This is the sequential tunneling regime. On the contrary, if inelastic scattering events are absent or rare, the junction is in the coherent tunneling regime. Second, electrons at the spacer feel Coulomb repulsions among them, whose strength is quantified by the energy $U$. Then, if $U>\Gamma_{R}$, the energy spectra at the spacer can not be represented by a simple one-electron picture and the junction usually enters the Coulomb blockade regime.

\section{MICROELECTRONICS: LOGICS AND SENSING CAPABILITIES}

The purpose of this section is to review the different physical effects that can be found in two-terminal experiments. These are experiments where one connects a piece of a metal to an external power, which supplies a voltage $V$ across the circuit such as the one sketched in Fig. 4(a). The intensity measured is plotted in Fig. 5(a). The figure shows that Ohm's linear relationship $I=V / R$, where $R$ is the resistance of the material, is verified only for low to moderate voltages. For high voltages, where $I$ depends non-linearly on the voltage, it is still possible to define the differential resistance $R_{d}=d V / d I$.

A third electrode, called the gate electrode, is sometimes placed close to the device. The purpose of the gate electrode is to subject the sample to a transverse (gate) voltage which changes the amount of current that flows from source to drain. Any current flowing from the gate to the sample is usually an undesired effect. Therefore the device is designed to prevent it. 

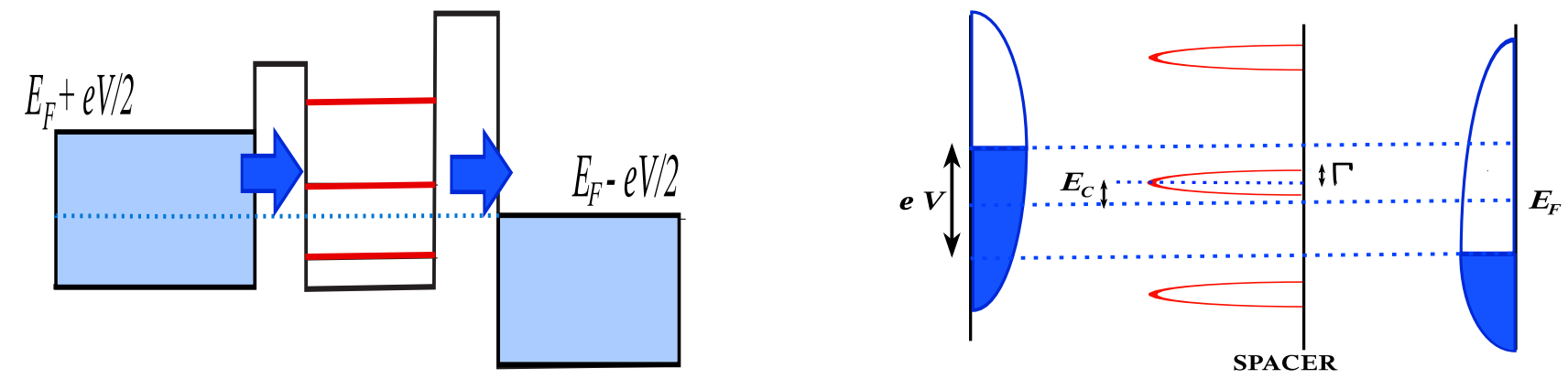

FIG. 4: (Color online) Left panel: sketch of the potential landscape and the energy spectrum of a molecular junction or quantum dot. The Fermi left and right electrodes ; right panel: plot of the densities of states at the electrodes and at the spacer.

An intrinsic semiconductor or an insulator of gap $E_{G}$ does not conduct any current until the voltage $V$ reaches $E_{G}$. This value is manifested by a sharp rise in $I(V)$, as we show in Fig. 5(b). A semiconductor may be doped with impurities that supply electrons or accept them (e.g.: supply holes, which are positively charged particles). We say that the semiconductor is $\mathrm{n}$ or $\mathrm{p}$ type, respectively. A doped semiconductor conducts a small but finite current for voltages smaller than $V$, which depends non-trivially on the impurity concentration. This current is provided by the extra electrons or holes supplied by the impurities.

By joining an n-type and a p-type semiconductor, a pn junction is fabricated. This device, also called a diode, shows the interesting behavior shown in Fig. 5(c). The diode effect is such that the device only conducts for positive biases, larger than $V_{T}$. If an $\mathrm{AC}$ voltage is supplied to a diode, the negative values of the current are filtered out, as shown in figure 6 . This effect, called rectification, is the most important functionality that diodes have in microelectronics. Actually, rectification consists of turning an AC signal into a DC current, and can be simply achieved with a circuit of a few diodes.

Possibly the single most important device in microelectronics is the Field Effect Transistor (FET), that we show schematically in Fig. 4(b). A FET is the basic building block of a chip. It consists of two n-type semiconductors (drain and source) deposited on a p-type semiconducting matrix, and separated by a narrow conducting strip called the channel. An insulating layer first and then metallic finger are placed on top of it. The insulating layer, called gate oxide, prevents the flow of leakage currents from the gate to the source. Possibly the most critical parameters in a FET are the channel length, and the thickness of the gate oxide.

Application of a voltage $V$ leads first to a ohmic region and for higher voltages to a saturation region. The most important effect of the FET though is its response to a gate voltage: a gate voltage shifts the $I V$ characteristics up or down, as we show in Fig. 5(d).

A switch is an electronic device that can provide two different values of the electrical current (typically a low value and a high value, well separated between them). The switch is referred to as a "gate" when these two values are abstracted to the mathematical form of boolean logic, so that one of them is assigned to 0 and the other to 1 . The use of electronic gates to function as a system of logical gates is the fundamental basis for the CPU unit of a computer, i.e. a CPU is a system composed of a huge number of electronic switches which function as logical gates.

Many different logical gates are realized in a computer. One of the simplest is the OR gate. An OR gate, depicted in Fig. $7(\mathrm{a})$, is a switch that receives two or more inputs and produces only one output. The output is 1 if at least one of the inputs is 1 , and zero only if all inputs are zero. The AND gate, depicted in Fig. 7(b), also has at least two inputs and one output. In this case the output is 1 only if all inputs are 1 , and is 0 otherwise. A NOT gate outputs $1(0)$ if the input is $0(1)$.

A Dynamic Random Access Memory (DRAM) is composed of many cells that hold individual bits of information. The heart of each cell is a capacitor that can store electrical charge. A capacitor can be in two states, charged or discharged, and therefore stores ' 1 ' and ' 0 ' bits of information. The plates of the capacitor are separated by an oxide dielectrics, like $\mathrm{SiO}_{2}$, or $\mathrm{Ta}_{2} \mathrm{O}_{5}$. This oxide should have a dielectric constant as large as possible to prevent dielectric breakdown, since the electric fields between the two plates can be extremely large as the size of the cell shrink. The capacitors lose charge very fast, and must be recharged about one thousand times per second.

Solid State electronic transport can be used not only to build computers, but also to make other important devices like sensors for instance. An electronic-based sensor exploits the fact that the electrical current in a solid-state device is modified under changes in the external environmental parameters, like pressure, temperature, magnetic field, light, or chemical identity of the molecules impinging and getting attached to the device. The critical parameters in a sensor are its sensitivity and specially its selectivity. Of course a sensor must be sturdy enough to withstand and provide reliable data under varying and frequently adverse ambient conditions. The coming years will possible witness a huge increase in the development and usage of wireless sensors, e.g.: sensors that pick data, perform simple analysis and transmit them to a central processing unit. 

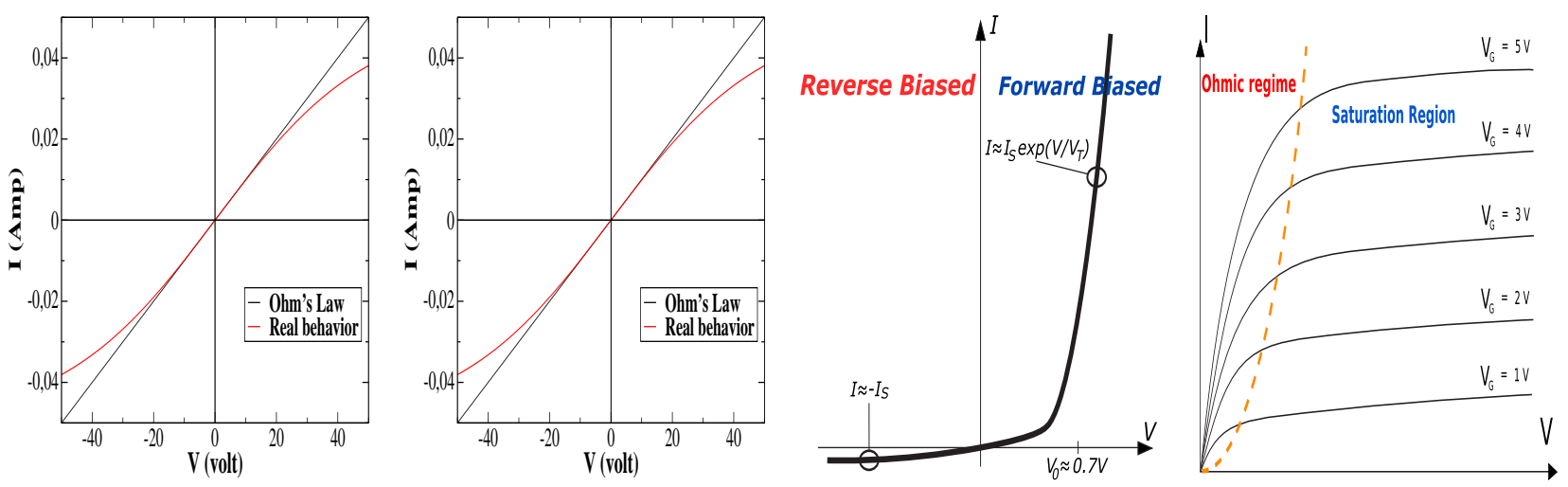

FIG. 5: (Color online) $I V$ characteristics (e.g.: a plot of the current $I$ as a function of the voltage drop $V$ ) of (a) a metallic sample of resistance $\mathrm{R}$; (b) a semiconductor of gap $E_{G}$; (c) a diode; (d) a FET.

\section{THE (SLOW) DEATH OF MOORE LAW}

Moore Law, the fact that the number of transistors on a single integrated circuit chip roughly doubles every 18 months has been the main driver of progress in today's Information and Communications Society. A roughly similar law holds for other key computer components, such as the DRAM memory. Moore's law is usually rephrased graphically in the feature size of a chip or DRAM component (typically, the channel length, or the side of the capacitor plate, respectively), as shown in Fig. 8, and Table I. Current technology is reaching its physical limits for a number of reasons. One limitation has to do with the wavelength of light, which governs the minimum feature size that can be imprinted by lithography. This is currently at $0.4-0.7$ micrometers, and will possibly be reduced to 1 micrometer, but no much further than that. A second problem has to do with the power losses that every FET in a chip produce. These losses are generated by the leakage of currents between the gate and the drain of the FET. Current leakage is prevented by the oxide layer barrier separating the gate and the channel. But as this gets thinner and thinner (the current thickness is of about 1.2 nanometers, see Table I), electrons can tunnel from one side to the other at an ever increasing rate. This generates a terrible heat management problem as well as an impaired signal to noise ratio. A third problem relates to the dielectric breakdown in DRAM memory capacitors. The shrinking area of the capacitor plates requires also a reduction in the thickness of the dielectric spacer, currently at about 1 nanometer. This is turn leads to electric fields larger than the maximum field that a material can withstand, which is of order $10^{7} \mathrm{~V} / \mathrm{cm}$.

There are basically three ways in which current technology can improve. One is the vertical approach, which aims at decreasing the size of the electronic components. Another is the horizontal approach, which looks for better designs and cleverer electronic components for a given size. The third one would correspond to a radical new approach based on new ideas and components like molecules or quantum computation. Extremely clever designs are pushing the limits of to- day's CMOS technology ever further, since as Gordon Moore put it, No Exponential is forever... but we can delay 'forever'. But there is no doubt that in the end the venerable CMOS micro and even "Nanoelectronics" technology must give way to radically new approaches, that will be based in quantum physics and chemistry, like graphene electronics, or molecular electronics.

As in every business matter, the demise of a long-standing main actor in the play, and the rise of new stars must be viewed not as a catastrophic event, but rather as the scenario where a new wealth of opportunities will arise. For instance, organic molecules with tailored shapes and functionalities can be made and assembled with the techniques of synthetic chemistry to realize ultracompact molecular integrated circuits. This fascinating and extremely powerful bottom-up approach is expected to decrease the minimum feature size of electronic devices even below the $1 \mathrm{~nm}$ mark, giving rise to an increase of orders of magnitude in the number of devices that can be packed in a chip. The same tunneling quantum effect that produces heating in today's computers, can be used to produce the main signal in a molecular FET, thereby reducing heating effects. Ultrafast memories can be fabricated by the use of molecules where precise control of the switching between two molecular states can be achieved by external means. Boolean logic gates can furthermore be fabricated that do not use the FET concept. Furthermore, precise control of the electronic states in a molecule should allow the replacement of Boolean by quantum logic, where extremely more powerful algorithms should lead to an exponential increase in the processing power of computers. The performance of the all-important contacts between a logic device and the circuitry in the chip will ultimately be controlled to unimaginable levels by suitable chemical bond engineering. The low performance of semiconductor optoelectronics will be possibly overhauled by dedicated circuits where optically active molecules have been tailored to either receive or emit electromagnetic radiation of given wavelengths. 


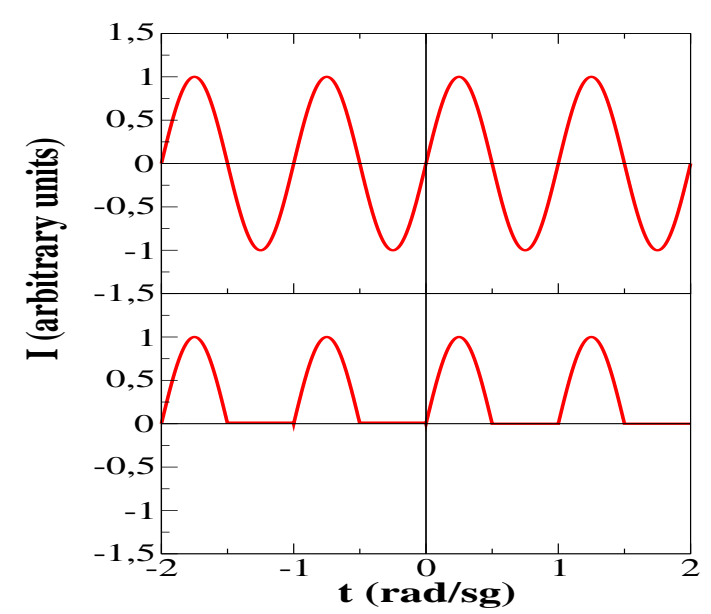

FIG. 6: (Color online) Top panel: AC current as a function of time; bottom panel: rectification provided by a diode.

\section{SPINTRONICS, MEMORY AND LOGICS}

We will present in this section basic notions about the physical effects or concepts behind spintronics. These are the notion of a ferromagnet, the degree of spin polarization, the magnetic anisotropy and the spin-flip mean free path. We will finally come to discuss the heart of spintronics, namely the anisotropic magnetoresistance (AMR) and giant magnetoresistance (GMR) effects.

\section{What is a ferromagnet?}

A ferromagnet is a material that displays a spontaneous macroscopic magnetization. To understand the physics behind this spontaneous magnetization, we will introduce a simple model that consists of an atomic chain that has a single orbital per atom. Its electronic structure is displayed in Fig. 9(a), and assumes that the electronic bands $\epsilon_{k, \sigma}$ for up and down spins are exchange-split by an energy amount $\Delta_{k}=\epsilon_{k, \uparrow}-\epsilon_{k, \downarrow}$ which, for this model does not depend on the $k$-vector. The magnetization is generated by the different densities of electrons $n_{\sigma}$ occupying up and down spin states in the material, and can be estimated using the formula

\begin{tabular}{|c|c|c|c|c|c|}
\hline & 1999 & 2003 & 2006 & 2009 & 2012 \\
\hline DRAM (half-pitch, $\mu$ m) & 0.18 & 0.13 & 0.10 & 0.07 & 0.05 \\
\hline DRAM samples, Gbit & 1 & 4 & 16 & 64 & 256 \\
\hline MPU (gate length, $\mu \mathrm{m}$ ) & 0.14 & 0.10 & 0.07 & 0.05 & 0.035 \\
\hline Transistors M MPUs $/ \mathrm{cm}^{2}$ & 6.2 & 18 & 39 & 84 & 180 \\
\hline Voltage $(\mathrm{V})$ & $1.5-1.8$ & $1.2-1.5$ & $0.9-1.2$ & $0.6-0.9$ & $0.5-0.6$ \\
\hline
\end{tabular}

TABLE I: Feature size and number of elements in DRAM and CPU chips as a function of year.

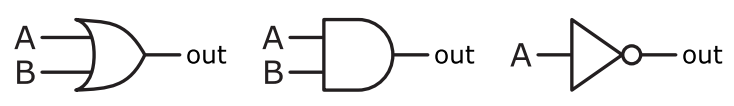

FIG. 7: (Color online) Some of the simplest logical gates, from left to right: OR, AND and NOT.

$$
M=-\mu_{\mathrm{B}}\left(n_{\uparrow}-n_{\downarrow}\right)
$$

where $\mu_{\mathrm{B}}$ is the Bohr magneton. To find $n_{\sigma}$ one must simply count the number of occupied states for each band, and divide by the total volume $V$. Since there are more $\uparrow$-spin than $\downarrow$-spin electrons, they are called majority and minority spin electrons, respectively. A cartoon of the spin-up and -down densities of states $g_{\uparrow, \downarrow}$ is shown in Fig. 9(b) where it is shown how they are split by $\Delta_{k}$. It is important to stress that the majority spin component need not have a higher $g_{\sigma}$. Indeed, the opposite happens in the specific case depicted in Fig. 9(b).

Magnetic materials are usually made of the $3 \mathrm{~d}$ transition metals $\mathrm{Fe}, \mathrm{Co}$ or $\mathrm{Ni}$, or some of their alloys. These materials have a complex electronic structure, since the corresponding atoms have both the $4 \mathrm{~s}$ and the $3 \mathrm{~d}$ shells unfilled. Their ground state atomic configuration is $4 \mathrm{~s}^{2} 3 \mathrm{~d}^{n}, n=6,7,8$, which leads to atomic spin moments $M$ of 4, 3 and 2 Bohr magnetons, respectively. Both s- and d-electrons in bulk Fe, $\mathrm{Co}$ and $\mathrm{Ni}$ take part in the chemical bonding, but in a somewhat different manner because of the different spatial extent of the orbitals. This is illustrated in Fig. 9(c), where we plot the band structure of Ni. Notice first that the s-band is wide, as expected for very delocalized orbitals, but also that it is only half-filled. This means that approximately 1 electron has been transferred to the d-band orbitals. Further, the s-band is not spin-split, which means that it does not participate at all in the magnetism of the material. The 5 bands of the $d$ complex are much narrower however, which reflects the shorter extension of the d-orbitals, and spin-split by approximately $1 \mathrm{eV}$. Notice that the majority ( $\uparrow$ spin) bands are fully occupied and have their highest point at about $1 \mathrm{eV}$ below the Fermi energy. The minority bands are on the contrary partly occupied by 4 electrons, since they have to make room for the electron transferred from the s-orbital. The spin moment of nickel corresponds therefore to a $\mathrm{d}^{9}$ atomic configuration, so that it can have a maximum value of $1 \mu_{\mathrm{B}}$. This value is further reduced to $0.62 \mu_{\mathrm{B}}$ because of the partial delocalization of the d-electrons. A similar argumentation holds for Fe and Co.

\section{The spin polarization}

The specific electronic structure of the ferromagnet has also important consequences for electronic transport, which is determined, as discussed in section 1 by quantities evaluated at the Fermi energy, see formula (6). For nickel, the majorityspin bands are fully occupied and do not participate in the electronic transport. In contrast, the spin-unpolarized s-bands 


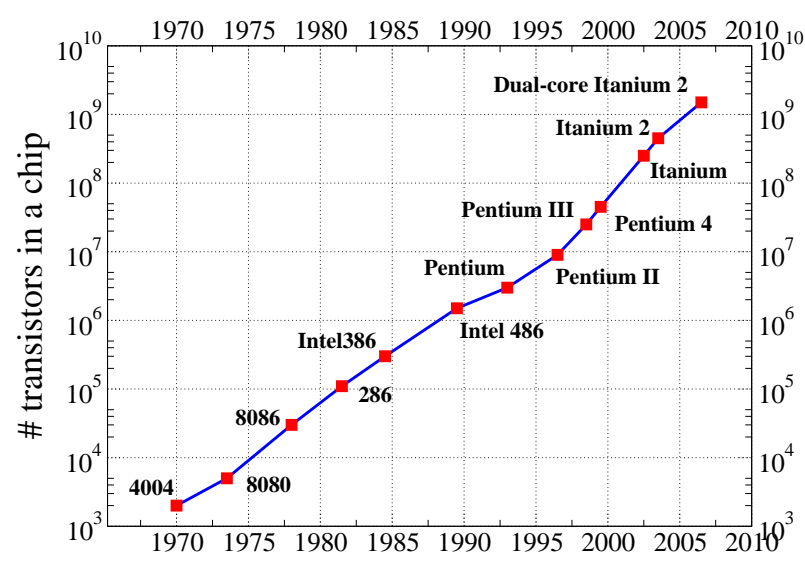

FIG. 8: (Color online) Time evolution of the minimum feature size of a FET

and the top of the minority $\downarrow$-spin d-band complex cross the Fermi energy. As a consequence, the electronic transport has a spin-unpolarized component due to the s-band and a spinpolarized component due to the minority-spin bands. Therefore the spin character of the electronic transport is opposite to that of the magnetization.

A useful tool to quantify the degree of polarization of the current density that a material provides is the Bulk Spin Polarization, defined as

$$
P_{2}=\frac{j_{\uparrow}-j_{\downarrow}}{j_{\uparrow}+j_{\downarrow}} \simeq \frac{N_{\mathrm{F} \uparrow} v_{\mathrm{F} \uparrow}^{2}-N_{\mathrm{F} \downarrow} v_{\mathrm{F} \downarrow}^{2}}{N_{\mathrm{F} \uparrow} v_{\mathrm{F} \uparrow}^{2}+N_{\mathrm{F} \downarrow} v_{\mathrm{F} \downarrow}^{2}}
$$

where we have assumed the same relaxation time for both spins. This magnitude provides a rough indication of the potential that a material may have to act as an spin injector, e.g.: a source of spins. Detailed calculations show that the the bulk polarization of nickel is basically zero, while that of iron is equal to 0.2 . Clearly enough, it is very important to maximize $P_{2}$, which is achieved by eliminating unpolarized s- or p- bands and leaving only majority- or minority-spin bands at the Fermi energy.

A material fulfilling this property is obviously called a halfmetal: it is metallic but has only one spin component at the Fermi level. The remarkable properties of half-metals were proposed in 1983 by de Groot and coworkers[17]. Typical half-metallic materials are manganite quaternary compounds, of which $\mathrm{La}_{0.67} \mathrm{Ca}_{0.33} \mathrm{MnO}_{3}$ is an example. These compounds are paramagnetic insulators at room temperature however and only become half-metals below the critical temperature $T \sim 220 \mathrm{~K}$. The compound $\mathrm{CrO}_{2}$ is a better candidate for spintronics applications since it is a half-metal with a Curie temperature of about $400 \mathrm{~K}$.

A spin-injection experiment is typically performed by joining a ferromagnet to another material, which can be metallic or semiconducting. The ability of the ferromagnet to inject spins in the other material depends not only on the Bulk Spin Polarization, but also on the details of the interface between the two materials. Injection of spins is therefore better characterized by the Junction Spin polarization $P_{J}$, which can be quantified by[18]

$$
P_{J}=\frac{N_{\mathrm{F} \uparrow} v_{\mathrm{F} \uparrow}^{n}-N_{\mathrm{F} \downarrow} v_{\mathrm{F} \downarrow}^{n}}{N_{\mathrm{F} \uparrow} v_{\mathrm{F} \uparrow}^{n}+N_{\mathrm{F} \downarrow} v_{\mathrm{F} \downarrow}^{n}}
$$

where $n$ can be any number from zero to two. $P_{J}$ can actually be even a more complicated function in many cases, since it depends on details such as the nature of both materials, the surface reconstruction, the presence of an oxide layer, the amount of disorder and the chemical bonding between the atoms at either side. In general the injection of spins in a junction must be dealt with case by case and $P_{2}$ provides a quick indication of the efficiency of the junction, which usually overestimates the actual $P_{J}$.

\section{Magnetic anisotropy}

The energy of any sample of a magnetic material has a contribution which depends on the orientation of the magnetization vector $\vec{M}=M \vec{\Omega}_{\mathrm{M}}$ with respect to the crystallographic axes and the external shape of the sample, $E_{\mathrm{MA}}\left(\vec{\Omega}_{\mathrm{M}}\right)[19]$. This magnetic anisotropy energy, although small, is usually high enough to keep $\vec{\Omega}_{\mathrm{M}}$ pinned to one of the important axes of the sample, and is therefore called magnetic anisotropy energy (MAE). In a experiment, $\overrightarrow{\Omega_{\mathrm{M}}}$ can be forced to rotate from one direction to another by applying a rotating magnetic field $\vec{H}$ of a magnitude higher than a threshold value, of the order of hundreds of Gauss; therefore the MAE also depends on $\vec{H}$, $E_{\mathrm{MA}}\left(\vec{\Omega}_{\mathrm{M}}, \vec{H}\right)$.

The part of the MAE which depends on the shape of the sample is called the shape anisotropy, and arises from the magnetic dipolar interactions. $E_{\text {shape }}$ is of the order of 0.1 $\mathrm{meV} /$ atom for bulk samples of iron, cobalt and nickel. The other part of the MAE, which depends on the orientation of $\vec{\Omega}_{\mathrm{M}}$ with respect to the crystallographic axes, is called magnetocrystalline energy, and is controlled by the Spin-Orbit contribution to the Hamiltonian $H=V_{S O} \vec{L} \cdot \vec{S}$, where $V \sim 80$ $\mathrm{meV}$ for $\mathrm{Fe}, \mathrm{Co}$ and $\mathrm{Ni}$. Since $V$ is much smaller than the average width $W$ of the bands in a ferromagnet, $E_{\text {crystal }}$ can be computed by perturbation theory. Further, the first three terms in the perturbative expansion cancel because of the symmetries of a bulk sample and $E_{\text {crystal }} \simeq\left(V_{S O} / W\right)^{3} V_{S O} \sim$ $0.001 \mathrm{meV} /$ atom. Therefore the MAE of a bulk sample is controlled by the shape anisotropy. In contrast, in a thin layer or a small magnetic dot of microscopic size, the second order in perturbation theory does not cancel, since the symmetry of the system is reduced, and $E_{\text {crystal }} \simeq\left(V_{S O} / W\right) V_{S O} \sim 0.1$ $\mathrm{eV} /$ atom. This leads to a competition between both sources of anisotropy.

For instance, the magnetization of a thin film is controlled by the bulk contribution which, as stated above, is dominated by the shape anisotropy. This favors the alignment of $\omega_{M}$ with the film plane (this is called easy plane magnetization). 

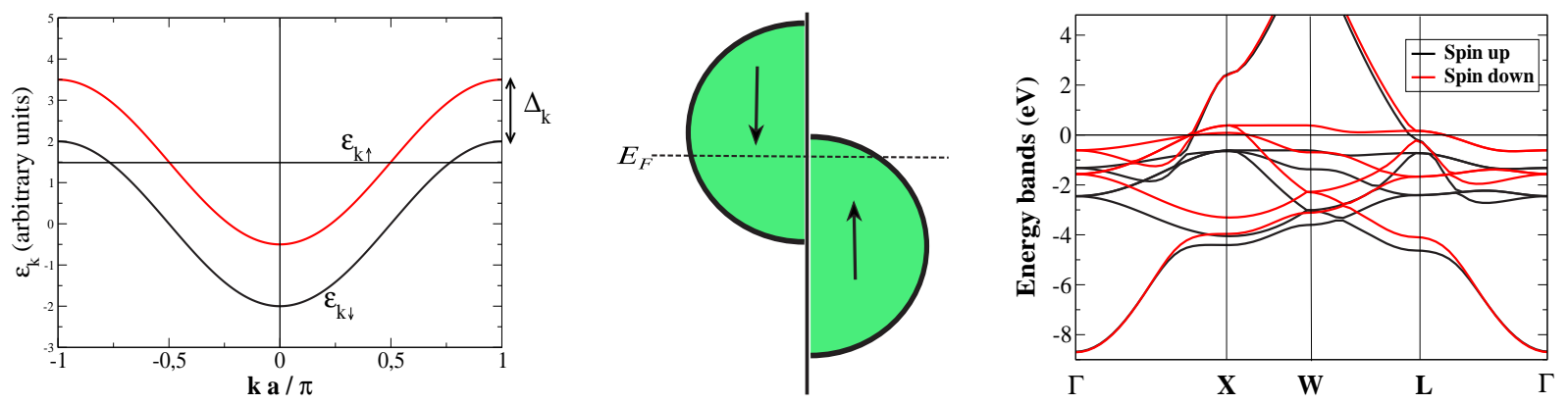

FIG. 9: (Color online) Left panel: band structure of a linear chain of atoms, that has one orbital per site; middle panel: Spin-polarized densities of states of the chain; right panel: band structure of fcc nickel.
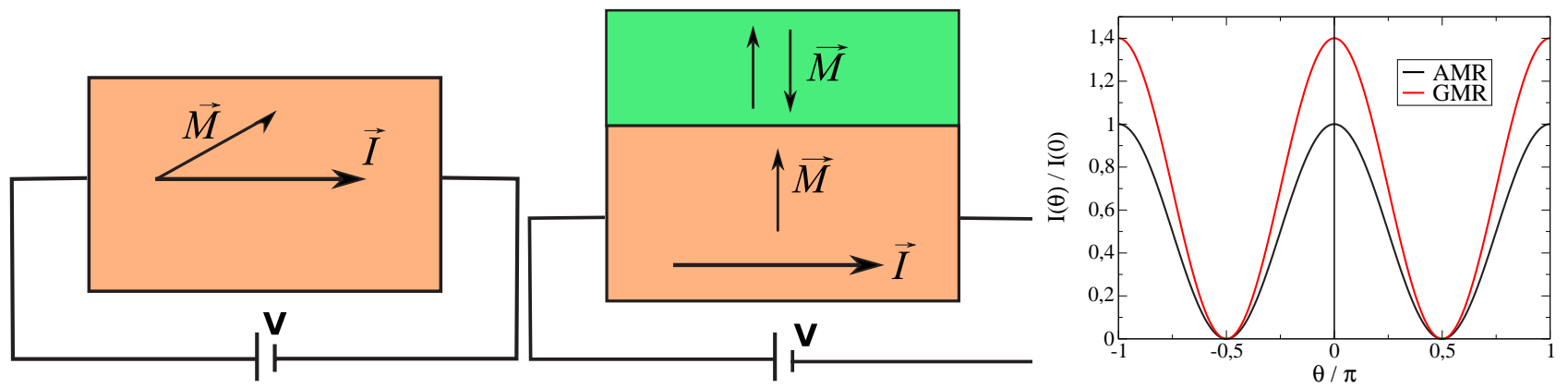

FIG. 10: (Color online) (a) A ferromagnetic material in the presence of a magnetic field that reorients the magnetization vector $\vec{M}=M \Omega_{\mathrm{M}}$, attached to an external supply. (b) A ferromagnetic bilayer coupled antiferromangetically, attached to an external power supply in the CIP geometry. (c) read head on top of a magnetic bit. The magnetization in the read head rotates an angle $\theta$ from the easy axis, due to the presence of the magnetic field originated by the magnetic bit; central panel: A GMR head on top of a magnetic bit; the magnetization in the blue ferromagnet rotates a larger angle $\theta$ than for the AMR head. right panel: the current flowing through the sample as a function of the angle for both heads.

The surface anisotropy energy $E_{S}$, which is controlled by the Spin-Orbit interaction, begins to compete with the bulk contribution for film thicknesses of the order of a few nanometers, and becomes dominant when the thickness is below about 1 nm. Further, $E_{S}$ can be negative or positive, depending on the surface under consideration. In the first case, it favors that $\omega_{M}$ align perpendicular to the film plane (easy axis magnetization), and the other way round. The magnetization of a thin film is therefore easy plane generically, but flips to easy axis for film thicknesses below $1 \mathrm{~nm}$.

The bits of information on the surface of a hard disk are magnetic dots of microscopic size and have a uniaxial MAE. Therefore $\vec{\Omega}_{\mathrm{M}}$ can only point in the two opposite directions of the easy anisotropy axis, and any rotation of $\vec{\Omega}_{\mathrm{M}}$ is prevented by the MAE barrier. Alas, this barrier gets reduced as the size of the bit decreases and eventually the bits enter the superparamagnetic limit, where the $E_{\mathrm{MA}}<K_{\mathrm{B}} T$ and the magnetization is free to rotate.

Interestingly enough, an atomic or molecular structure may have much fewer symmetries than a surface or bulk sample. Correspondingly, $E_{\text {crystal }}$ receives contributions from first order perturbation theory. Small $3 d$ and $4 d$ atomic clusters are therefore predicted to have anisotropies of the order of 10 to $50 \mathrm{meV}$ [20, 21], while the $5 \mathrm{~d} \mathrm{Ir}$ and Pt elements, where $V_{S O} \sim 500 \mathrm{meV}$, should make clusters or molecular struc- tures with anisotropies $E_{\mathrm{MAE}} \simeq 100-500 \mathrm{meV}$, which are expected to overcome the superparamagnetic limit[20]. As a summary, Ir- and Pt- based molecular structures are predicted to function as true nanomagnetic bits of information.

\section{Spin-flip mean free path}

Spintronics is currently used to make memory devices, but it also holds the promise to lead to low-power logics devices. The fabrication of spintronics logic gates assumes the ability to create tailored spin-polarized currents, as well as to preserve their spin-polarization for lengths and times long enough. However, a spin-polarized current will lose its character if the spins of the electrons flip due to some sort of scattering event. The average length then that an electron traverses before its spin is reversed is called the spin-flip mean free path $l_{\text {sf }}$.

The spin of an electron in a bulk sample is not conserved (e.g.: may be flipped) if the material presents non-collinear magnetism in the form of spin spirals, or, more typically, whenever there are domain walls, since in this case the electron will feel a non-uniform spin-dependent potential landscape.

For a conventional ferromagnetic material, the spin land- 
scape is fully collinear, which means that the spins only point upwards or downwards. But still an electron traversing the materials can flip its spin due to the Spin-Orbit interaction. This happens because the Spin-Orbit Hamiltonian has two terms that raise or lower the $z$-component of the spin, and therefore is non-diagonal in the spin components,

$$
H_{\mathrm{SO}}=V_{\mathrm{SO}}\left(L_{z} S_{z}+\frac{1}{2}\left(L_{+} S_{-}+L_{-} S_{+}\right)\right)
$$

Spin flip events via the Spin-Orbit interaction are activated by lattice vibrations, which modify locally the term $\vec{L}$ in the Hamiltonian. The size of these lattice vibrations can be quantified by the number of phonons in the material $n_{p h}(T)[9]$. Since this number increases with temperature, so does the spin flip events with the concomitant decrease of $l_{\mathrm{sf}}$.

The spin flip mean free path is typically of the order of several hundred nanometers in many materials. Ideally, $l_{\mathrm{sf}}$ should be larger than the size of the system, which can indeed be achieved in nanostructures like carbon nanotubes or metallic nanowires, where the spin mean free path at low temperatures has indeed been measured to be of the order of hundreds of nanometers [22, 23].

When $l_{\mathrm{sf}}$ is indeed of the order or even larger than the size of a sample, the surface, or better to say, the contacts become a relevant source of spin flip scattering. Indeed, the contacts of a junction are frequently spin-active, since they contain spin-flip scattering centers which can alter significantly the low voltage, low temperature IV characteristics of the sample The ultimate spin-logics devices will be fabricated when delicate control of the spin state of each individual electron be attained, so that it can be kept or flipped at will.

Finally, while the Spin-Orbit interaction is an essential ingredient in the fabrication of molecular magnetic bits, its influence is detrimental for molecular logic. Hence, one should use heavy elements like Ir or Pt to fabricate molecular bits, while light elements such as carbon are preferred for logic functionalities, such as spin valves.

\section{Anisotropic magnetoresistance versus giant magnetoresistance}

Imagine that an external magnetic field is applied to a ferromagnetic device connected to a external supply as in Fig. 10(a). Anisotropic magnetoresistance (AMR) is the effect whereby the two-terminal current $I$ depends on the angle $\theta$ that the magnetic field (and therefore the magnetization $\vec{\Omega}_{\mathrm{M}}$ of the sample) makes with the direction of the current.

The Giant Magnetoresistance (GMR) is a related, but different, effect [24, 25, 26]. Imagine that two ferromagnetic samples $\mathrm{A}$ and $\mathrm{B}$ are placed together in a bilayer configuration. Furthermore, suppose that sample A is connected to an external power supply as depicted in Fig. 10(b). This geometry is called CIP, for current in plane, since the interface between both ferromagnets lies parallel to the direction of current flow. Imagine that sample A has the magnetization orientation $\vec{\Omega}_{\mathrm{A}}$
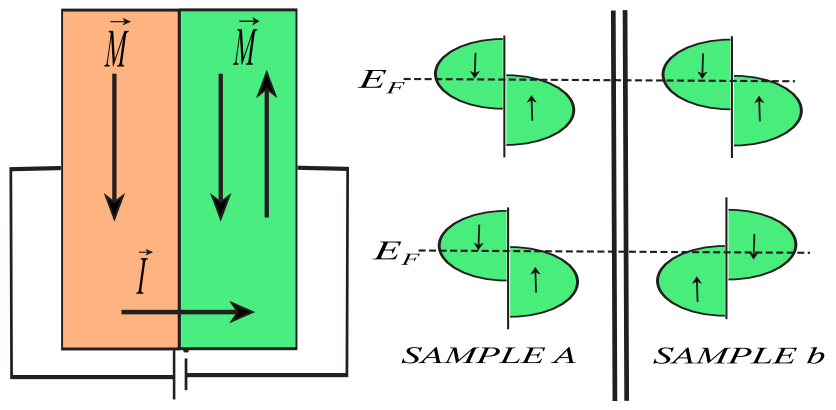

FIG. 11: (Color online) (a) The GMR-CPP geometry. A sketch of the spin-polarized DOS of samples $\mathrm{A}$ and $\mathrm{B}$, when both magnetizations are parallel (b) and perpendicular (c).

pinned by some means. Then the magnetization of sample B $\vec{\Omega}_{\mathrm{B}}$ is aligned antiparallel to $\vec{\Omega}_{\mathrm{A}}$, if no magnetic field is applied to the device. The effect that produces this alignment is called exchange coupling, and its strength is measured by the exchange energy constant $J$, which is usually of the order of a few hundred of Gauss. The magnetization $\vec{\Omega}_{\mathrm{B}}$ can be flipped to be parallel to $\vec{\Omega}_{\mathrm{A}}$ and back, by turning on and off a magnetic field parallel to $\vec{\Omega}_{\mathrm{A}}$ of strength larger than $J$. Even though the power supply is attached to sample A, the current $I$ flows through the whole bilayer and senses whether both magnetizations are aligned or not. In the first case, the spin polarized electrons suffer fewer scattering events when crossing the interface, or travelling through sample $\mathrm{B}$, than in the second. Therefore the current is higher in the first case than in the second. The variation of the current in the CIP geometry is larger than in the AMR effect (hence the name Giant) for two reasons. First, the angle made by the rotating magnetization is larger, $180^{\circ}$; second there are more scattering events in the CIP geometry than in the AMR geometry.

A third possibility exists, where the variation of the intensity is enhanced further. It consists of placing the bilayer perpendicular to the direction of flow of the current, as shown in Fig. 11(a). This geometry is called current perpendicular to plane (CPP), and the design is called a spin valve. In this case, all the spin-polarized electrons that leave sample A are fed into sample B. The reason for the enhancement is a simple bottleneck effect for the flow of the spin-polarized currents. When $\vec{\Omega}_{\mathrm{A}}$ and $\vec{\Omega}_{\mathrm{B}}$ are parallel, the Densities of States (DOS) for both majority and minority components of the spin are equal, as shown in Fig 11(b). On the contrary when $\vec{\Omega}_{\mathrm{A}}$ and $\vec{\Omega}_{\mathrm{B}}$ are antiparallel, the electrons of the minority component in sample A suffer a bottleneck effect when trying to enter sample B.

The quality of a GMR device is defined by the ratio

$$
G M R=\frac{I_{\text {parallel }}-I_{\text {antiparallel }}}{I_{\text {antiparallel }}}
$$

which is called the (optimistic) GMR ratio. The pessimistic definition substitutes $I_{\text {antiparallel }}$ by $I_{\text {parallel }}$ in the denominator of the equation. Here $I_{\text {parallel }}\left(I_{\text {antiparallel }}\right)$ denote the low 

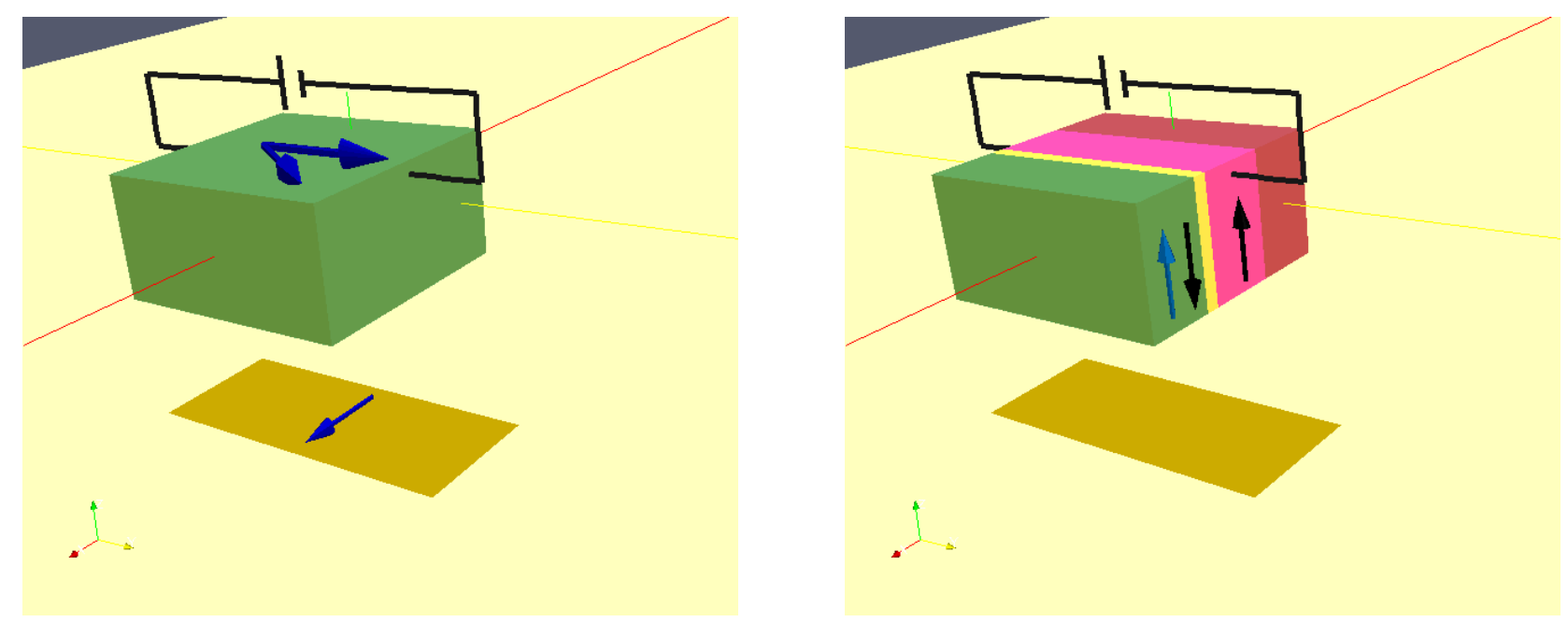

FIG. 12: (Color online) Left panel: An AMR read head on top of a magnetic bit. The magnetization in the read head rotates an angle $\theta$ from the easy axis, due to the presence of the magnetic field originated by the magnetic bit; right panel: A GMR head on top of a magnetic bit; the magnetization in the blue ferromagnet rotates a larger angle $\theta$ than for the AMR head.

voltage intensity current when both ferromagnetic samples are aligned parallel (antiparallel) to each other.

It is possible to design magnetic field sensors by exploiting the sensitivity of the current to the external magnetic field in any of the three geometries discussed above. Indeed, the technology behind the reading head in old hard disk drives is based on the AMR effect, while the new ones are based on the GMR-CIP geometry. A hard drive consists of an array of microscopic magnetic dots. These have a high magnetic anisotropy so that their magnetization is aligned along the easy-plane axis, which accounts for the two memory states, 0 and 1 . Therefore each dot creates a magnetic field according to its magnetization. The read head scans the array so that it feels the magnetic field of each dot via the magnetoresistive effect when its is positioned above it. The magnetic fields produced by the dots determine then the current that passes through the head. Typical read heads, are shown in Fig. 12, while a very nice animation showing their behavior can be found in [27]. The GMR head has four slabs: the two ferromagnetic materials are separated by an insulator. The fourth slab is an antiferromagnetic material which is used to pin the orientation of the magnetization of the first ferromagnet by the exchange bias effect[28, 29].

\section{Electric control of the magnetization}

The write head in a hard drive is a coil whose size is much larger than the read head. Whenever a magnetic dot is required to reverse the orientation of its magnetization, an electric pulse passes through the coil, which originates a magnetic field high enough to force the reversal of the magnetization in the dot. Downscaling a hard drive therefore also requires finding an alternative mechanism to reverse the magnetization of the dots. Much research is devoted to finding low-power, effective, all- electrical mechanisms that force this reversal. One possibility is to use the GMR effect in the CPP geometry, whereby the head would have the pinning antiferromagnet and the pinned ferromagnet, while the magnetic bits in the surface of the hard disk would become the second ferromagnet. Since now the spacer between the two ferromagnets is substituted by vacuum space, the effect is called tunneling magnetoresistance (TMR). Normal operation of the head as a read head would then use the TMR-CPP effect. The application of a DC current density (therefore a DC voltage) above a critical threshold would allow the reversal of the magnetization of the bit via the spin-torque effect[30, 31], therefore enabling the write operation. This approach has the important drawback that electrical currents flow through the surface of the hard disk drive that must be drained, and which can lead to spurious charging effects throughout the surface.

A promising approach consists of storing domain walls in magnetic nanowires[32]. A domain wall pattern in a nanowire can encode tens or hundreds of bits, which can be read via the TMR effect, and written via the motion of the domain walls based on the spin-torque effect. Since magnetic nanowires can be packed in two dimensional patterns or even three dimensional arrangements, this approach could lead to memory devices as fast as DRAM memories, and as cheap as hard disk drives[32].

\section{MOLECULAR ELECTRONICS}

\section{A brief introduction}

Traditionally the field of molecular electronics was initiated by Aviram and Ratner, who studied the rectifying properties of a donor-acceptor molecular bridge [16]. From an experimental point of view the electronic properties of molecules 
have been studied mainly by using the scanning tunneling microscopy (STM) [33, 34] and the mechanically controllable break junction (MCBJ) [35, 36]. In the STM case the molecules are bonded to a surface (traditionally gold) with a certain coupling atom (traditionally sulphur or nitrogen) so that they stay upright or with some degree of tilting. The STM tip is then used to contact them on the opposite side and measure their transport properties. Other approaches are based on positioning the tip on a certain place of the surface and waiting until molecules attach to it $(I(t)$ technique) [37]. It is also possible to attach gold nanoparticles [38] on the other end to make the contact configuration more reproducible or contacting the STM tip to the gold surface and pulling it away in a solution that contains the molecules [39]. In the MCBJ technique the molecules are fist chemisorbed on the surface of the tips and then these are brought together until a molecule makes contact to both tips. It is also possible to use electromigration to separate the tips [40], which allows to gate the molecules [41, 42]. Slightly different methods include the atomic force microscopy (AFM) [43], micro fabricated structures [44], crossed wires geometries [45] and using a mercury drop to make the contact [46].

While great success has been achieved with such techniques, they have the handicap that they are not scalable, i.e. they do not allow to integrate thousands of molecules in a single chip in an easy an reproducible way. Also, the main experimental problem all these techniques have is related to the contact geometry, which is not clearly defined and can vary from measurement to measurement. This uncertainty leads to two types of fluctuations in the measurements: one due to the irreproducibility of values obtained at different times on the same molecule and the other due to variations between molecules. For that reason it is necessary to make as many measurements as possible and collect the values in an histogram.

Conceptually there are various transport regimes that can characterize these systems. In small molecules the transport is ballistic, which means the dimensions of the molecule are smaller than the electron mean free path, as opposed to diffusive transport. Depending on wether the electron conserves its energy when it passes through the junction or exchanges energy with other particles such as phonons, the transport can be elastic or inelastic. Attending to the coupling of the molecule to the contacts $(\Gamma)$ and the energy difference between the Fermi level and the frontier molecular orbital $\left(E_{\mathrm{G}}\right)$ it is possible to distinguish four transport regimes [47]. If both $\Gamma$ and $E_{\mathrm{G}}$ are small, transport is characterized by sequential tunneling (Coulomb blockade). If the coupling increases the strong interaction of electrons with the molecule can lead to polaronic transport in long molecules and almost transparent behavior in short molecules. For low coupling but high $E_{\mathrm{G}}$ the junction is in the tunneling regime, where electrons don't 'see' the molecule. Finally, for high $E_{\mathrm{G}}$ and $\Gamma$, transport is in the Landauer regime, characterized by resonances in the transmission probabilities which signal the molecular levels in the junction. These resonances are called Breit-Wigner resonances [48] and are Lorentzians whose width is related to the

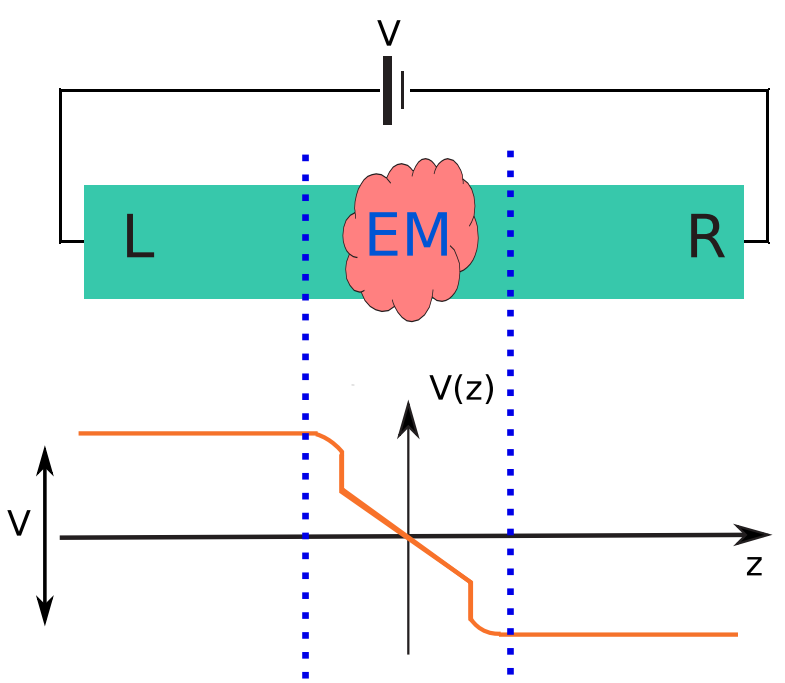

FIG. 13: (Color online) Sketch of the partitioning scheme, where the system is divided in three pieces.

coupling to the contacts $(\Gamma)$ (these are not however the only ones, other types are possible like Fano resonances [49, 50]). This last regime is the most popular from a theoretical point of view because it allows to use ab-initio techniques to calculate the transport properties consistently.

\section{Caroli's approach to molecular electronics}

The most popular technique used to simulate the transport properties of a molecular junction is the so-called nonequilibrium Green's function formalism (NEGF). The technique is based on the non-equilibrium formalism of statistical mechanics introduced by Keldysh [51, 52]. The NEGF formalism for transport was introduced by Caroli and coworkers [53], and popularized by Datta [8]. It uses the partitioning scheme sketched in Fig. 13, whereby the whole system in Fig. 1(a) is divided in three chunks: left electrode (L), right electrode $(\mathrm{R})$ and the extended spacer or extended molecule (EM). Notice that the electric potential and the electronic structure of a portion of the electrodes close to the contacts is modified since it feels the proximity of the electrode's surface and of the molecule, as well as the potential drop at the contacts. We illustrate this effect as a bending of the electrical potential profile in Fig. 13. The extended molecule hence comprises the molecule and part of the $\mathrm{R}$ and $\mathrm{L}$ electrodes. The criterion to make the mathematical cleavage is that the $\mathrm{L}$ and $\mathrm{R}$ electrodes must act as thermodynamic reservoirs of electrons and therefore must display exactly the electronic structure of a bulk sample of the same material. Ferrer and coworkers[13] proposed and alternative scheme where the system is partitioned in only two pieces, the molecule becoming attached to one of the two electrodes.

The NEGF technique can be combined with Time Dependent Density Functional Theory[54] (TDDFT). This is the 


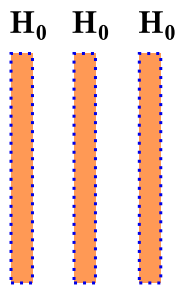

$\begin{array}{lll}\mathrm{H}_{1} & \mathrm{H}_{1} & \mathrm{H}_{1}\end{array}$

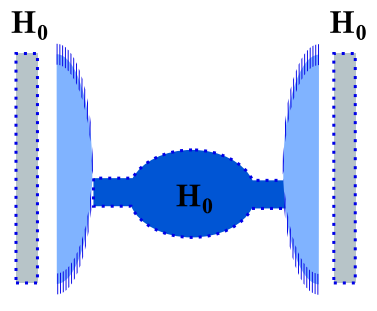

EM

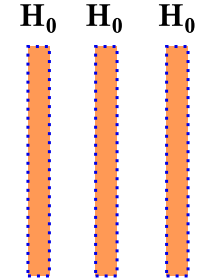

$\begin{array}{lll}\mathrm{H}_{1} & \mathrm{H}_{1} & \mathrm{H}_{1}\end{array}$

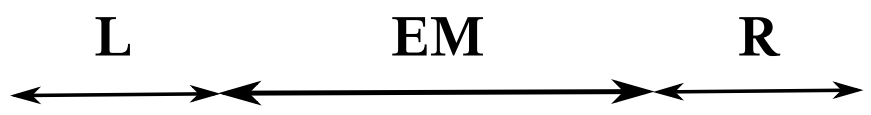

FIG. 14: (Color online) Schematic view of the molecular junction, where the $\mathrm{L}$ and $\mathrm{R}$ electrodes have been decomposed into Principal Layers.

method of choice of many groups, which adapt an ab-initio electronic structure code to obtain self-consistently the Nonequilibrium Green's functions of the system, which in turn are used to compute the charge density $n(\vec{r})$ and the current through, say, the left contact $I_{L M}$. Notice that the current passing any surface perpendicular to the direction of electron flow is the same since charge must be conserved. Therefore, we will denote the electronic current as $I$ henceforth. While detailed accounts of the NEGF can be found in textbooks[8] and many research papers[55, 56, 57], we believe it worth it to include here a brief exposition.

\section{Explicit description of the NEGF in a localized atomic basis set}

We suppose that the eigenstates of the system of Fig. 13 can be expanded in terms of a basis set of localized atomic orbitals $\psi_{\mu}\left(\vec{r}-\vec{R}_{\mu}\right)=<\vec{r} \mid \psi_{\mu}>$, where $\mu=n, l, m$ denote the radial and angular quantum numbers of the orbital and $\vec{R}_{\mu}$ denotes its position:

$$
\left|\phi_{i}>=\sum_{\mu} c_{\mu}(i)\right| \psi_{\mu}>
$$

The Hamiltonian matrix elements can be written in this ba- sis set as $H_{\mu, \nu}=<\psi_{\mu}|H| \psi_{\nu}>$, while $S_{\mu, \nu}=<\psi_{\mu} \mid \psi_{\nu}>$ describes the overlap between two given orbitals.

We define now a Principal Layer (PL) as the smallest cell in the electrodes that repeats periodically in the direction of the transport, such that it has non-zero Hamiltonian matrix elements only with its nearest-neighbor Principal Layer. We then decompose both electrodes in PL, as depicted in Fig. 14. Notice also that the extended molecule also contains at least one PL at each side. Let us assume that each PL has $N$ orbitals, while EM contains $M$ orbitals, where $M$ must of course be larger than $2 \mathrm{~N}$.

We then define

- $h_{0}$ as the $N \times N$ matrix containing all Hamiltonian matrix elements $H_{\mu, \nu}$ where both $\mu$ and $\nu$ belong to the same Principal Layer.

- $h_{ \pm 1}$ as the $N \times N$ matrix containing the matrix elements $H_{\mu, \nu}$ where $\mu$ and $\nu$ belong to two nearest-neighbor Principal Layers.

- $h_{\mathrm{LM}}$ is an $N \times M$ matrix whose matrix elements $h_{\mu, \nu}$ are such that $\mu$ is an orbital in $\mathrm{L}$ and $\nu$ is an orbital located in M. $h_{\mathrm{ML}}=h_{\mathrm{LM}}^{+} \cdot h_{\mathrm{RM}}$ is an $N \times M$ matrix whose matrix elements $H_{\mu, \nu}$ are such that $\mu$ is an orbital in $\mathrm{R}$ and $\nu$ is an orbital located in M. $h_{\mathrm{MR}}=h_{\mathrm{RM}}^{+}$.

- $H_{\mathrm{M}}$ is an $M \times M$ matrix where both $\mu, \nu$ belong to the extended molecule.

and employ similar definitions for the overlap matrices. Notice that the matrices $h_{\mathrm{LM}}$ and $h_{\mathrm{RM}}$ couple the electrodes to the extended molecule. Because we have included PLs at the edges of the extended molecule, they have the following simple expression:

$$
h_{\mathrm{LM}}=\left(\begin{array}{lll}
h_{1} & 0 & \ldots
\end{array}\right) ; \quad h_{\mathrm{RM}}=\left(\begin{array}{lll}
\ldots & 0 & h_{1}
\end{array}\right)
$$

Then the Hamiltonian can be expressed in this basis, when the system is at zero voltage, as

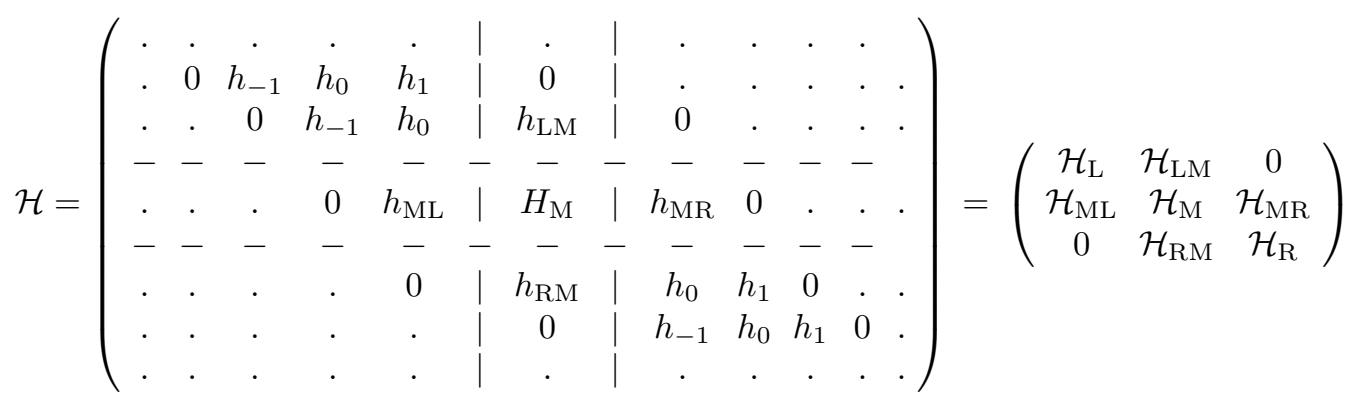

Application of the voltage $V$ drives the system out of equilibrium. The Hamiltonian is modified to 


$$
\mathcal{H}=\left(\begin{array}{ccc}
\mathcal{H}_{\mathrm{L}}+e V_{L} \mathcal{S}_{\mathrm{L}} & \mathcal{H}_{\mathrm{LM}}+e V_{L} \mathcal{S}_{\mathrm{LM}} & 0 \\
\mathcal{H}_{\mathrm{ML}}+e V_{L} \mathcal{S}_{\mathrm{ML}} & H_{\mathrm{M}} & \mathcal{H}_{\mathrm{MR}}+e V_{R} \mathcal{S}_{\mathrm{MR}} \\
0 & \mathcal{H}_{\mathrm{RM}}+e V_{R} \mathcal{S}_{\mathrm{RM}} & \mathcal{H}_{\mathrm{R}}+e V_{R} \mathcal{S}_{\mathrm{R}}
\end{array}\right)
$$
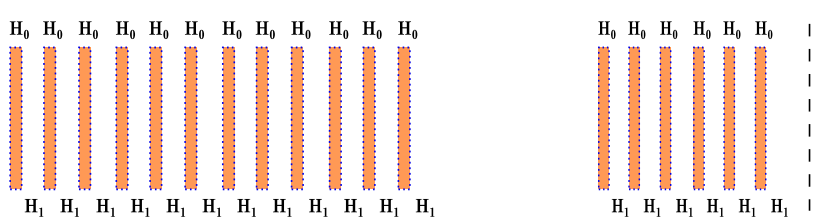

FIG. 15: (Color online) Left panel: schematic view of the infinite auxiliary system that is used to compute $H_{0}$ and $H_{1}$. Right panel: sketch of the auxiliary semi-infinite system employed to compute $\mathcal{G}_{\mathrm{L}}^{\mathrm{OR}}$.

where $V_{L, R}= \pm V / 2$.

Notice that the partition of the system has been chosen so that $h_{0}$ and $h_{1}, s_{0}$ and $s_{1}$ are not influenced at all by the presence of the contact or the molecule. Therefore, they can be computed separately using the infinite system depicted in Fig. 15(a), before performing the actual simulation of the system of Fig. 14. As a consequence, all of the sub-matrices of $\mathcal{H}$ are known a priori except for $H_{\mathrm{M}}[n(\vec{r})]$. This sub-matrix depends on the details of the extended molecule and also on the voltage bias $V$. Furthermore, notice that in Density Functional Theory the Hamiltonian depends self-consistently on the charge density

$$
n(\vec{r})=\sum \rho_{\mu \nu} \psi_{\mu}(\vec{r}) \psi_{\nu}(\vec{r})
$$

The density matrix $\rho_{\mu \nu}$ can be simply computed as a sum over all the eigenvalues $i$ of the system,

$$
\rho_{\mu \nu}=\sum_{i} c_{\mu}(i) c_{\nu}^{*}(i) g(i)
$$

where $g(i)$ is the occupation factor of each eigenstate, which in equilibrium is given by the Fermi distribution $f(i)$. The problem here is that the occupation of each state is no longer given by $f(i)$ when the voltage $V$ is applied. This problem can nevertheless be solved using the NEGF formalism, with the help of the retarded, advanced and Keldysh matrix Green functions $\mathcal{G}^{R}, \mathcal{G}^{A}$ and $\mathcal{G}^{<}$(that we will describe below). These Green's functions can be decomposed similarly to the Hamiltonian as

$$
\left(\begin{array}{ccc}
\mathcal{G}_{\mathrm{L}} & \mathcal{G}_{\mathrm{LM}} & \mathcal{G}_{\mathrm{LR}} \\
\mathcal{G}_{\mathrm{ML}} & G_{\mathrm{M}} & \mathcal{G}_{\mathrm{MR}} \\
\mathcal{G}_{\mathrm{RL}} & \mathcal{G}_{\mathrm{RM}} & \mathcal{G}_{\mathrm{R}}
\end{array}\right)
$$

where the superscripts $R, A$ and $<$ have been omitted for simplicity. Then, the density matrix for a given pair of orbitals can be expressed with the more general formula

$$
\rho_{\mu \nu}=\frac{1}{2 \pi i} \int d E G_{\mu \nu}^{<}(E)
$$

which reduces to formula (22) in equilibrium conditions.

The computation of the charge density $n(\vec{r})$ at the extended molecule is a step that can not be avoided if one wishes to simulate transport properties. To show this, we come back to the expression of the Hamiltonian $\mathcal{H}$ in formula (20) and notice that $H_{\mathrm{M}}$ can be Gaussian-eliminated so that the Hamiltonian is reduced to

$$
\mathcal{H}=\left(\begin{array}{cc}
\mathcal{H}_{\mathrm{L}}^{\prime}+e V_{L} \mathcal{S}_{\mathrm{L}}^{\prime} & \mathcal{T}\left(H_{\mathrm{M}}\right) \\
\mathcal{T}^{+}\left(H_{\mathrm{M}}\right) & \mathcal{H}_{\mathrm{R}}^{\prime}+e V_{R} \mathcal{S}_{\mathrm{R}}^{\prime}
\end{array}\right)
$$

Clearly, the current can be written in terms of the trace of $\mathcal{T} \mathcal{T}^{+}$. Since this matrix can be diagonalized, its diagonal elements can be understood as the contribution of each conducting channel. This line of reasoning, while very appealing, is unpractical since $\mathcal{T}$ can only be computed explicitly if $H_{\mathrm{M}}$ is known. The way to proceed then is exactly the opposite: eliminate from the Hamiltonian $\mathcal{H}_{L, R}$, which are known a priori.

The Keldysh formalism supplies the following matrix equations for the retarded Green's function:

$$
[\epsilon \mathcal{S}-\mathcal{H}] \mathcal{G}^{\mathrm{R}}(E)=\mathcal{I}
$$

where $\epsilon=E+i \delta$, and $E$ and $\delta$ are the energy and an infinitesimal number. By Gaussian elimination, one finds that

$$
G_{\mathrm{M}}^{\mathrm{R}}(E)=\left[\epsilon S_{\mathrm{M}}-H_{\mathrm{M}}-\Sigma_{\mathrm{L}}^{\mathrm{R}}(E)-\Sigma_{\mathrm{R}}^{\mathrm{R}}(E)\right]^{-1},
$$

where we have introduced the retarded self-energies $\Sigma_{\mathrm{L}, \mathrm{R}}^{R}$. which account for the effects of the electrodes onto the leads:

$$
\Sigma_{\mathrm{L}}^{\mathrm{R}}(E)=\left(\epsilon_{L} S_{\mathrm{ML}}-H_{\mathrm{ML}}\right) G_{\mathrm{L}}^{0 \mathrm{R}}(E)\left(\epsilon_{L} S_{\mathrm{LM}}-H_{\mathrm{LM}}\right)
$$

and

$$
\Sigma_{\mathrm{R}}^{\mathrm{R}}(E)=\left(\epsilon_{R} S_{\mathrm{MR}}-H_{\mathrm{MR}}\right) G_{\mathrm{R}}^{0 \mathrm{R}}(E)\left(\epsilon_{R} S_{\mathrm{RM}}-H_{\mathrm{RM}}\right) .
$$

where $\epsilon_{L, R}=\epsilon+e V_{L, R}$. The surface Green's functions $\mathcal{G}_{\mathrm{L}, \mathrm{R}}^{0 \mathrm{R}}(E)$ are equal to

$$
\left[\epsilon_{L, R} \mathcal{S}_{\mathrm{L}, \mathrm{R}}-\mathcal{H}_{\mathrm{L}, \mathrm{R}}\right]^{-1}
$$


Notice that $\mathcal{G}_{\mathrm{L}}^{0 \mathrm{R}}(E)$ is called a surface Green's functions since it corresponds to the Hamiltonian $\mathcal{H}_{\mathrm{L}}$, which describes only to the semi-infinite left electrode, severed mathematically from the rest as shown in Fig. 15(b). And the same can be said of $\mathcal{G}_{\mathrm{R}}^{\mathrm{OR}}(E)$. The calculation of these surface Green's functions is in many cases numerically complicated, and astute algorithms must be devised[55, 58]

The Keldysh Green's function can be obtained from the following matrix equation

$$
\mathcal{G}^{<}=\mathcal{G}^{\mathrm{R}}\left[\mathcal{G}^{0 \mathrm{R}}\right]^{-1} \mathcal{G}^{0<}\left[\mathcal{G}^{0 \mathrm{~A}}\right]^{-1} \mathcal{G}^{\mathrm{R}}
$$

In particular, the Keldysh Green's function for the extended molecule is

$$
\begin{aligned}
& G_{\mathrm{M}}^{<}(E)= \\
& i G_{\mathrm{M}}^{\mathrm{R}}(E)\left[\Gamma_{\mathrm{L}}(E) f\left(E-e V_{L}\right)+\Gamma_{\mathrm{R}}(E) f\left(E-e V_{R}\right)\right] G_{\mathrm{M}}^{\mathrm{R} \dagger}(
\end{aligned}
$$

where the line-widths

$$
\Gamma_{\mathrm{L}, \mathrm{R}}(E)=i\left[\Sigma_{\mathrm{L}, \mathrm{R}}^{\mathrm{R}}(E)-\Sigma_{\mathrm{L}, \mathrm{R}}^{\mathrm{A}}(E)\right]
$$

From this equation, one computes the density matrix at the extended molecule using equation (24). The current that crosses the left or the right contacts can be written in terms of the transmission coefficient

$$
T(E)=\operatorname{Tr}\left[\Gamma_{\mathrm{L}} G_{\mathrm{M}}^{\mathrm{R} \dagger} \Gamma_{\mathrm{R}} G_{\mathrm{M}}^{\mathrm{R}}\right]
$$

by the expression

$$
I=\frac{e}{h} \int d E T(E)\left(f\left(E-e V_{L}\right)-f\left(E-V_{R}\right)\right)
$$

The self-consistency procedure that is employed to perform transport calculations is similar, but not identical, to that used in vanilla Density Functional Theory. One first computes and sets apart $h_{0}$ and $h_{1}$ using the geometry in Fig. 15 (a). One can then compute the surface Green's functions $\mathcal{G}_{\mathrm{L}, \mathrm{R}}^{0 \mathrm{R}}(E)$ using equation (30) and the geometry in Fig. 15(b). These are used to compute the self-energies $\Sigma_{\mathrm{L}, \mathrm{R}}^{R, A}$, using Eq. (29) and the line-widths $\Gamma_{\mathrm{L}, \mathrm{R}}$ from Eq. (33). Notice that these magnitudes do not depend on the density matrix at the extended molecule $\rho_{\mathrm{M}}$ so that they can be computed just once before the self-consistency procedure actually starts, and be stored[59].

The selfconsistency procedure starts with a trial $\rho_{\text {old }}$ which is used to compute the charge density $n(\vec{r})$ at the molecule via Eq. (21). The charge density is in turn employed to compute the Hamiltonian $H_{\mathrm{M}}$, which serves to compute $G_{\mathrm{M}}^{\mathrm{R}}$ via Eq. (27). The retarded Green function $G_{\mathrm{M}}^{\mathrm{R}}(E)$, together with the line-widths $\Gamma$ is finally used to compute the Keldysh Green's function at the molecule $G_{\mathrm{M}}^{<}$via Eq. (32). Integrating this function in energy yields a new density matrix $\rho_{\text {new }}$, Eq. (24). The procedure starts again by updating $\rho_{\text {old }}=\rho_{\text {new }}$. The cycle stops when $\left|\rho_{\text {new }}-\rho_{\text {old }}\right|$ is smaller than a required tolerance parameter. Once a converged density matrix is achieved, the current $I$ is computed via Eqs. (34) and (35).

\section{Accuracy of the method and improvements}

As commented above, Time Dependent Density Functional Theory [54] is the formalism which integrates the Keldysh formalism with Density Functional Theory to account for nonequilibrium phenomena. TDDFT theory is implemented in practice in NEGF by using the lowest order approximation possible, which amounts to computing the Hamiltonians $h_{0}$, $h_{1}$ and $H_{\mathrm{M}}$ with the vanilla recipes of Density Functional Theory[60, 61]. The most widely used implementations of the NEGF method indeed rely on the local density approximation (LDA) [62], the generalized gradient approximation (GGA)[63], or the BLYP scheme[64] to compute the exchange and correlation functional. Exact exchange or hybrid functionals have been scarcely used in molecular electron$E)$ and GGA approximations in any case provide usually qualitatively correct results even when the junction is in the tunneling regime, where strong electron correlations can be important. The calculations tend to underestimate the HOMOLUMO gap and to predict a somewhat incorrect alignment of the Fermi-level. Additionall, they err sometimes in the prediction of the most stable contact geometries or the inter-atomic distances.

More specifically, the transport properties of molecules strongly bonded to the electrodes agree qualitatively but not quantitatively with experiments [35], with both the low-bias gap and absolute value of the current and conductance too high [65, 66]. However, the agreement is getting better as the codes and theory improve and excluding a few cases (like the archetypical Au-BDT junction) the differences between theory and experiment are within one order of magnitude [67]. The correspondence between theory and experiment at low biases can also be improved by a certain choice of the coupling atoms and the Fermi level [69], which shows again the importance of these factors. In general, most calculations prove that the agreement increases as the molecules become longer, since the relevance of the coupling to the leads and the electronic correlations decrease with the length of the molecules.

The most established method to include correlations beyond LDA is based on the inclusion of self-interaction corrections as proposed by Perdew and Zunger[62] (SIC-PZ). Notice that LDA and GGA erroneously include the Coulomb interaction of each electronic eigenstate with itself. This leads for instance to the incorrect prediction that the energy of the ground state of the hydrogen atom is $\sim-7 \mathrm{eV}$ instead of the correct value $(-13.6 \mathrm{eV})$. Inclusion of the SIC-PZ correction fully corrects this error. This scheme can easily be applied to single atoms, but is extremely costly numerically for the case of molecules or solids. A few years ago, Filippeti and Spalding [70] proposed a series of approximations to the SICPZ scheme that rendered it numerically acceptable. While the Filipetti-Spalding scheme relies on a series of uncontrolled approximations, Sanvito and coworkers[71, 72] have implemented it in NEGF and shown that it improves substantially 
the predictions for the conductance of short molecular junctions. Ke and coworkers[73] have also studied the effects of SIC corrections on the conductance of molecular junctions.

The SIC scheme is by itself not a strongly correlated approach. On the contrary, it removes part of the electron correlations that are overestimated by LDA and GGA. Higher orders in TDDFT have been included by Sai and coworkers [74]. A numerically costly but quite accurate approximation to strong correlations is the GW scheme, which has been explored in NEGF by Darancet and coworkers, and Thygesen and Rubio[75, 76].

It is quite difficult to make a complete account of the different methods that have been introduced by the many groups across the world that work in Non-equilibrium electron transport. While our purpose in this review is to explain in some detail the standard approach to NEGF transport, we comment very briefly now on some of the many other alternative approaches. Fisher and Lee, and Meir and Wingreen[77, 78] discussed long ago the inclusion of strong correlations in Caroli's approach, using model tight-binding Hamiltonians. There exist a sizeable number of other proposals for Nonequilibrium electron transport that use the Keldysh formalism but do not rely on Caroli's partition scheme[13, 79, 80]. Master equations can also be used, together with, for instance, Configuration-Interaction techniques[81, 82].

\section{A survey of some of the relevant developments}

We pass now to review a few of the important effects and applications that have appeared in the past few years.

Inelastic transport. The electron-phonon interaction in nanoscale systems can be used to determine the presence of molecules inside the junction, a technique which is known as inelastic electron tunneling spectroscopy (IETS) [47]. In some cases it can lead to a increase in the current, which is seen as a peak in the second derivative of the $\mathrm{I} / \mathrm{V}$ curves [68] and is due to electrons which are deflected into transmission resonances. In other cases it can lead to a reduction in the current, which is seen as a dip in the second derivative [83]. For example, when the coupling is strong the reduction is due to the reflection of electrons to regions with depleted charge [84]. Theoretically this topic has been addressed extensively and many approximations have employed to include the effect of phonons on the transport characteristics, see e.g. [85, 86, 87, 88, 89, 90, 91].

Rectification. There have already been a lot of works on rectification properties of molecules. There are basically three rectifying mechanisms that can lead to diode-type behaviors: the donor-insulator-acceptor mechanism due to inelastic tunneling [16], the energy mismatch between levels localized on different parts of the molecule [92] and the asymmetric position of the molecular levels with respect to the Fermi level of the leads [93, 94]. Experimentally, these systems have been largely studied [95, 96, 97, 98] but their rectifying properties are still poor.
Optoelectronics. Molecules offer also the possibility of interacting with light [99]. They can be used as current-light converters [100, 101], where a current produces a luminous signal, or light-current converters [102, 103, 104], where light modifies the transport properties of the junction.

Switches and logic gates. Switches can be considered the most basic components of memory and logic. There have already been many propositions of molecular switches [105]. Some studies have demonstrate that it is possible to produce memory effects in molecules embedded in selfassembled monolayers [106], where the state of the molecule can be changed from more conducting $(\mathrm{ON})$ to less conducting (OFF) depending on how well ordered is the matrix surrounding it. There have been many other experimental and theoretical studies on switching, which include the field regulation of the molecular conductance by charged surface atoms [107], conformation changes [108, 109, 110] and spin effects [111]. The combination of various switches gives rise to a logic gate, which performs the basic logical operations AND, OR and NOT. Such gates have already been realized experimentally by using molecules as the basic building blocks [112].

Field effect transistor (FET). Although the FET can also be included in the category of switches it is better to treat it as a separate issue due to its importance in the field of electronics. The real challenge in this case is the positioning of the gate electrode, which has to be close enough to the molecule to ensure the field is strong. However, transistors based on carbon nanotubes [113], monolayers of molecules [114] or single molecules [41, 42, 115] have already been fabricated, which opens the possibility of using molecules as the basic building blocks in future circuits.

Wires. In order to fabricate feasible molecular circuits the electronic elements of the circuit should be connected between them and with those of the external world with wires not much bigger than them. It would be then necessary to develop molecular wires that can perform such tasks. They should be as small and simple as possible, should have almost independent wire-length behavior and should be flexible and resilient. While there are many examples of conjugatedbased molecular wires [116], where the transport can be superexchange-mediated or thermally activated, the most interesting molecular wires at the moment are those based on small conjugated carbon chains like for example polyyne chains [117], which are expected to produce high conductance, almost length-independent behavior and ohmic $I V$ characteristics.

Negative differential resistance (NDR). Since the discovery of the NDR effect [118] lots of applications in the field of semiconductor physics have been found [119, 120], which include amplification, logic and analog to digital conversion. In the context of molecular electronics there are various types of mechanisms that can lead to the phenomenon of NDR. One, related again to the field of semiconductor physics, is due to the entrance of resonances in the bulk silicon gap as the bias is applied [121], which allows the possibility of tuning the 
FIG. 16: (Color online) $I V$ characteristic and Conductance of tunneling vs transparent regimes. NDR

NDR peak by varying the coupling between the STM tip and the molecule. Others include chemical changes [44], the destruction of conductance resonances as a consequence of the misalignment of localized states [122, 123] and local orbital symmetry matching [124].

Sensors. The use of molecular electronics systems as sensors is based on a simple principle. Whenever another molecule approaches the molecule in the junction the original molecular levels are shifted and distorted, which changes the current that flows along the device. It has already been proved that molecules are sensitive to environmental fluctuations [125, 126], such as the effect of water or other atmospheres. These studies suggest that molecules could be used as extremely quick, precise and tolerant sensors.

Heat transport and thermopower. Heat transport in nanoscale systems has already been measured [127], and addressed theoretically [128]. This issue is of paramount importance to determine the stability of the junction and the maximum currents that can flow before it melts. Another closely related issue is the thermopower [129], whose sign offers information of the position of the Fermi level relative to the molecular levels [130]. In fact molecules offer great possibilities to produce devices with huge thermopower characteristics and figures of merit if resonances are located near the Fermi level.

\section{MOLECULAR SPINTRONICS}

\section{Arquitectures}

The basic architecture of a molecular spintronics device must have two basic components, a molecule and some magnetic element, which can be the same molecule and/or the leads. If only the leads are (ferro)magnetic (F-PM-F) the system can act as a spin valve or a transistor. If on the other hand the molecule is magnetic (like for instance a molecular magnet) and the leads are non-magnetic (NM-MM-NM) the system can be used as a logic gate or as a quantum computation qbit. Finally, when all elements are magnetic (F-MM-F) the most important application relates to the magnetic bit. In the following we explain the basic characteristics and applications of each of these architectures.

F-PM-F. The possibility of using molecules between magnetic leads as spin-valves or transistors has already been demonstrated by many experiments [22, 131, 132, 133, 134] and predicted by several studies, either using semiempirical methods [135] or approximations to the magnetism in the leads [136, 137, 138]. The first ab-initio study which included realistic magnetic leads was carried out by the authors and coworkers [139]. The basic idea behind this application is that a molecule between magnetic leads can significantly alter the magnetoresistive ratio depending on how it couples to the minority and majority spin channels in the parallel and antiparallel configurations. In our study we used two types of molecules, 1,4-[3]-phenyl-dithiolate (1) and [8]-alkane-dithiolate (2). 1 is a conjugated molecule whose HOMO orbital is delocalized along the backbone and energetically near the Fermi level of the electrodes, which makes this molecule sort of metallic. In 2 however, all carbon bonds on the backbone are saturated, which moves the HOMO down in energy and depletes the backbone from delocalized electrons, so that this molecule is insulating. We found the magnetoresistive ratio (GMR) was dependant on the bias voltage and was different for each type of molecule. In this case the largest value was much higher for the metallic $\mathbf{1}(600 \%)$ than for the insulating $2(100 \%)$ molecule.

In general depending on the combination of molecules, coupling atoms and magnetic materials it is possible to have very interesting possibilities [140]. The worst scenario from a spintronics point of view happens if the transmission for the majority and minority spins is the same in the parallel and antiparallel configurations, which means the GMR is zero. If however the coupling to one type of electrons is zero (which means finite transmission in the parallel case and zero transmission in the antiparallel case) the GMR is infinite. It is also possible to have negative GMR when the total transmission in the antiparallel configuration is larger than that of the parallel configuration. Of course the total current in both configurations will always be smaller than the current obtained in the ideal case where the two magnetic materials are joined together without any scattering region, but what is interesting from the point of view of spintronics is the GMR ratio: the bigger the GMR the better the spin valve. Here lies the potential of molecular spintronics.

From a physical point of view, in order for these systems to be operative it is necessary the spin that enters the molecule does not flip, i.e. maintains its coherence, which means the molecule must be magnetically inert. The good news are that the two interactions that can lead to spin precession and decoherence, the spin-orbit and the hyperfine interaction, are very small in organic molecules. The spin orbit only becomes important for heavy elements and the hyperfine interaction in carbon (the main component of the organic molecules and where the frontier orbitals are usually localized) is zero because the nuclear spin in ${ }^{12} \mathrm{C}$ is null. Molecules are then very promising candidates for miniaturizing spintronics materials.

NM-MM-NM. In this case we have a single-molecule magnet (SMM) between non-magnetic leads [141]. Molecular magnets are a relatively new type of material where one or various magnetic atoms are embedded inside a organic molecule [142]. Most of the molecules with various magnetic atoms possess a high spin moment which can lead to interesting effects like quantum tunneling [143, 144] and interference due to the Berry phase [145]. The disadvantage of SMM is however their low anisotropic energy for reversal, which in most cases can be as low as various Kelvin. This makes them poor candidates to act as logic gates or devices for quantum com- 
putation.

Taking into account the physics, depending on the degree of coupling of the molecule to the leads it is possible to distinguish two regimes. In the weak coupling regime transport is dominated by Coulomb blockade and is therefore sequential. This means that an electron can hop into the molecule whenever a resonance coincides with the Fermi level but this electron forbids the hopping of another electron due to the charging energy $U$. The $I / V$ curve shows then a series of steps separated by $U$ or, taking into account the gate voltage, the typical Coulomb diamonds [41] in a 3D graph. By applying a magnetic field it is also possible to see spin transistor properties like non-linear behavior of the excitations as a function of the magnetic field [146]. If the molecule is strongly coupled to the leads the overall conductance increases significantly and, for small enough temperatures, it shows a peak at zero bias that splits under the presence of a magnetic field. Such peak is known as the Kondo peak and is produced by the coupling between the localized magnetic moment on the molecule and the conduction electrons in the leads [147, 148]. The Kondo effect has been seen in molecules with one [41] or two [42] magnetic centers. Both cases give rise to relatively high Kondo temperatures and in the last one the resonance can be tuned by the gate voltage and produce $\mathrm{ON}$ and OFF states [42].

The basic principle behind this logic gate is that the total current depends on the relative orientation of the spin in the molecule, which can be changed with a magnetic field or with a gate. In order for these devices to work is then necessary that they do not change spin state spontaneously at room temperature, i.e. they should have a high magnetic anisotropy, which means the spin-orbit coupling should be high. The magnetic anisotropy can depend also on how the magnetic ions are distributed and the distance between, but in general is not big enough to allow room temperature devices.

Finally, a very interesting application is related to quantum computation [149]. It has been predicted [150] that by using molecular magnets it is possible to implement Grover's algorithm for searching in databases [151]. Another possible use of the NM-MM-NM configuration could be related to the interaction between flying and static qubits [152]. However, the presence again of fluctuations and noise due to spin flips can make infeasible the design of such systems.

F-MM-F. This configuration combines the magnetic elements of both previous configurations and can lead to much richer phenomena than the other two, since it allows to define spin valves, transistors and logic gates. The most obvious application concerns the definition of bits, where the leads can be used to write and read the information and the molecule to store it. In that sense the second lead can be changed and substituted by a magnetic head to change the spin orientation or a conducting lead to read the information that is stored.

An example of this type configuration was proposed by encapsulating metallocenes inside carbon nanotubes [153]. Metallocenes are composed of two aromatic rings made of 5 carbon and five hydrogen atoms which sandwich a metallic element, typically a $3 d$ metal such as $\mathrm{V}, \mathrm{Cr}, \mathrm{Mn}, \mathrm{Fe}, \mathrm{Co}$ or $\mathrm{Ni}$. Depending on the type of element the molecule can be magnetic or not. For this series of metals the total magnetic moment varies as $3,2,1,0,1,2 \mu_{\mathrm{B}}$, which can be easily explained by taking into account the crystalline field produced by the aromatic rings and Hund's rules on the $d$ states of the metal. The metallocenes can only be encapsulated in a certain type of nanotubes [154] and tend to make chains inside of them. The chain produces a conduction channel inside the nanotube which adds to the conduction channels on the nanotube wall. If all spins in the chain are parallel the total number of channels at the Fermi level is 5. However, if one or various of the spins are reversed the channel on the metallocenes is killed and the total number of channels decreases to 4 , which gives a magnetoresistance ratio of $20 \%$. This provides then an example of a F-MM-F system that can be used for applications such as sensing, logic gates and bits and can also give rise to very interesting physical properties [155].

\section{Extending the NEGF to handle the spin degree of freedom}

Expanding the NEGF described above to include collinear magnetism and spin polarized currents is as straightforward as extending LDA to the local spin density approximation (LSDA): it is necessary just to attach the additional quantum number $\sigma=\uparrow, \downarrow$ to the eigenstates, density matrix, electron density, electric current, Hamiltonian and Green's functions. The eigenstates can therefore be written as

$$
\phi_{i, \sigma}(\vec{r})=\sum_{\mu} c_{\mu}(i, \sigma) \psi_{\mu}(\vec{r})
$$

Likewise, the electronic charge is written as

$$
n_{\sigma}(\vec{r})=\sum_{\mu, \nu} \rho_{\mu \nu, \sigma} \psi_{\mu}(\vec{r}) \psi_{\nu}(\vec{r})
$$

where the density matrix is

$$
\rho_{\mu \nu, \sigma}=\frac{1}{2 \pi i} \int d E G_{\mu \nu, \sigma}^{<}(E)
$$

and the Keldysh Green's function is

$$
G_{\mathrm{M}, \sigma}^{<}(E)=i G_{\mathrm{M}, \sigma}^{\mathrm{R}}(E)\left[\Gamma_{\mathrm{L}, \sigma}(E) f\left(E-e V_{L}\right)+\Gamma_{\mathrm{R}, \sigma}(E) f\left(E-e V_{R}\right)\right] G_{\mathrm{M}, \sigma}^{\mathrm{R} \dagger}(E)
$$


The transmission coefficients are also spin-dependent

$$
T_{\sigma}(E)=\operatorname{Tr}\left[\Gamma_{\mathrm{L}, \sigma} G_{\mathrm{M}, \sigma}^{\mathrm{R} \dagger} \Gamma_{\mathrm{R}, \sigma} G_{\mathrm{M}, \sigma}^{\mathrm{R}}\right]
$$

as well as the current

$$
I_{\sigma}=\frac{e}{h} \int d E T_{\sigma}(E)\left(f\left(E-e V_{L}\right)-f\left(E-V_{R}\right)\right)
$$

All the expression for the Green's functions are generalized by including the subindex $\sigma$. This simple extension allows to compute not only spin polarized currents in ferromagnetic structures, but also more sophisticated objects like spin valves.

The extension to non-collinear magnetism is slightly more complex. Generically, the eigenstates of the system are spinors of the form

$$
\tilde{\phi}_{i}(\vec{r})=\sum_{\mu} \tilde{c}_{\mu}(i) \psi_{\mu}(\vec{r})=\sum_{\mu}\left(\begin{array}{c}
c_{\mu \uparrow}(i) \\
c_{\mu \downarrow}(i)
\end{array}\right) \mid \psi_{\mu}>
$$

This spinor structure translates to the definition of electronic charge density which acquires now a $2 \times 2$ matrix structure,

$$
\tilde{n}(\vec{r})=\sum_{i} \tilde{\phi}_{i}(\vec{r}) \tilde{\phi}_{i}^{*}(\vec{r})=\left(\begin{array}{cc}
n^{\uparrow \uparrow} & n^{\uparrow \downarrow} \\
n^{\downarrow \uparrow} & n^{\downarrow \downarrow}
\end{array}\right)=\left(\begin{array}{cc}
n+m_{z} & m_{x}-i m_{y} \\
m_{x}+i m_{y} & n-m_{z}
\end{array}\right)=\sum_{\mu, \nu}\left(\begin{array}{cc}
\rho^{\uparrow \uparrow} & \rho^{\uparrow \downarrow} \\
\rho^{\downarrow \uparrow} & \rho^{\downarrow \downarrow}
\end{array}\right) \psi_{\mu}(\vec{r}) \psi_{\nu}(\vec{r})
$$

Notice that this $2 \times 2$ spin structure contains all the information required to determine both the electronic density $n(\vec{r})$ and the magnetization vector $\vec{M}(\vec{r})=\left(m_{x}, m_{y}, m_{z}\right)$. Further, the spin-matrix structure translates to all the Green's functions since,

$$
\rho_{\mu \nu}^{\sigma \sigma^{\prime}}=\frac{1}{2 \pi i} \int d E G_{\mu \nu}^{\sigma \sigma^{\prime}<}(E)
$$

Non-collinear magnetism is potentially important in molecular spintronics in two cases. The first relates to the simulation of spin valves. The GMR ratio in a spin valve was defined above as

$$
G M R=\frac{I_{\text {parallel }}-I_{\text {antiparallel }}}{I_{\text {antiparallel }}}
$$

One must therefore simulate two different magnetic configurations of the electrodes. In the first, the magnetization vector of the L and $\mathrm{R}$ electrodes is fixed to, say, the up-spin orientation; in the second configuration, the magnetization vector of the $\mathrm{R}$ electrode is swapped to the down-spin configuration. In this second configuration, the atomic magnetization must reverse its orientation somewhere in the extended molecule, forming a domain wall. This reversal of the magnetization occurs over a length $l_{D W}$. When $l_{D W}$ is long, $\vec{M}$ slowly turns around; when $l_{D W}$ is of the order of the interatomic spacing, $\vec{M}$ will just flip. In the first limit, the magnetic vector forms a non-collinear structure while the domain wall in the second limit is purely collinear. Bruno has argued that the length of a domain wall is of the order of its section[156]. A domain wall in an atomic contact or molecular junction is therefore expected to have a length of the order of the interatomic spacing, and to be collinear. We have indeed simulated a spin valve consisting of two nickel electrodes connected by a nickel chain of variable length between two and seven atoms, and found that the magnetization indeed flips abruptly and collinearly, and that this domain wall is nucleated at the contacts between the electrodes and the chain[157|.

The second case where non-collinear magnetism can be relevant is when the electronic structure and transport properties of the junction are influenced by the presence of heavy atoms. Indeed, heavy atoms have a strong Spin-Orbit interaction, whose Hamiltonian can be written in spin space as

$$
H_{\mathrm{SO}}=V_{S O}\left(\begin{array}{cc}
L_{z} & L_{x}-i L_{y} \\
L_{x}+i L_{y} & -L_{z}
\end{array}\right)
$$

The non-diagonal terms, besides giving rise to magnetic anisotropies, also flip the spin of the electrons producing a finite spin mean free path that can reduce the GMR ratio when $l_{\mathrm{sf}}$ is of the order of the length of the junction. Furthermore, the Spin-Orbit interaction alters the electronic structure in the junction and can open minigaps in a few cases. We have indeed found that these minigaps lead to a perfect GMR ratio in the case of infinite iridium and platinum zigzag chains[158].

There are only a few public NEGF ab-initio codes that can handle magnetic phenomena[56]. To the best of our knowledge, only our code Smeagol can also handle non-collinear structures. Furthermore, Smeagol includes self-consistently the Spin-Orbit interaction and therefore includes automatically in its simulations magnetic anisotropies, finite spin-flip mean free paths [20, 159], and other interesting phenomena.

\section{Examples of simulations}

The first calculations of transport properties of molecular electronics systems were carried out by a series of groups that used various types of approximations to include magnetic 


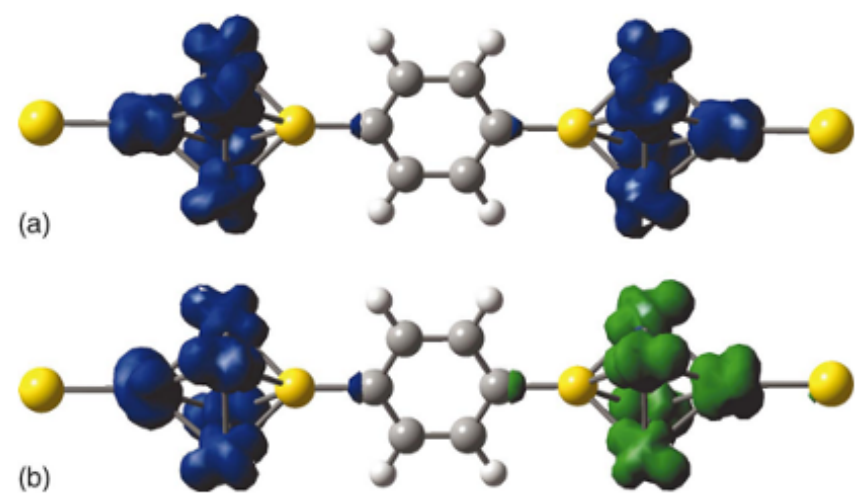

FIG. 17: (Color online) Example of system studied by Pati and coworkers [137], where they plot the electron spin density in the parallel (a) and antiparallel (b) cases.

leads or spin degrees of freedom. Emberly and Kirczenow used a semiempirical approach to predict for the first time the presence of GMR effects in molecules contacted between Ni electrodes [135]. Pati and coworkers studied the transport properties of carbon chains sandwiched between two cobalt atoms and coupled to non-magnetic leads and found a spin valve effect and a non-monotonic variation of the magnetoconductance as a function of the chain length [136]. The same group considered also the transport properties of a BDT molecule between Ni cluster layers and connected again to nonmagnetic leads where they found another GMR effect [137]. Wei and coworkers studied a carbon chain sandwiched between aluminium electrodes subjected to a magnetic fields at different relative orientations and found also a spin-valve effect [138].

The previous approximations to include the spin degree of freedom were necessary due to the high difficulty in calculating the surface Green's function of magnetic leads, which is used to obtain the self-energies. Such problem can be significantly reduced by using a semi-analytical method [160] that improves significantly over recursive algorithms [8, 161]. This allowed to calculate, as stated above, entirely from first principles the transport properties of metallic and insulating molecules between nickel electrodes and predict the appearance of GMR effects when the relative orientation of the magnetization of the leads was reversed. Similar studies were also used to calculate the magnetic and transport properties of nickel point contacts [55]. These calculations were done with the Smeagol code [55], which uses the Hamiltonian provided by the DFT code SIESTA [163] to calculate the transport properties of nanoscale systems using the NEGF formalism. It works by substituting the diagonalization of the Hamiltonian by the calculation of the Green's function of the extended molecule. This is necessary to fully simulate a semiinfinite system (on both electrodes) and to properly calculate the density matrix under a finite bias voltage.

Examples of spintronics calculations performed with Smeagol include molecular spin valves [139, 165], magnetic
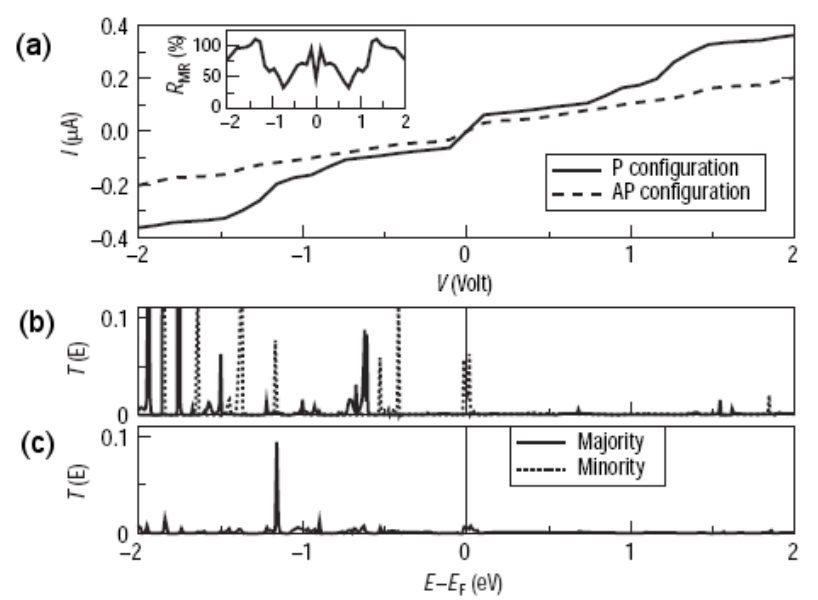

FIG. 18: (Color online) Transport properties of an octane molecule sandwiched between Ni leads. We plot the current as a function of the bias voltage (a) and the majority and minority transmission coefficients for the parallel and antiparallel configurations (b) and (c). The inset shows the magnetoresistive ratio as a function of bias voltage. Figure taken from [139].

nickel contacts [55, 166] and metallocenes encapsulated in carbon nanotubes [153]. In the first case, as stated previously, we studied the transport properties of two types of molecules, octane (8-alkane-dithiolate, insulating) and tricene (1,4-3-phenyl-dithiolate, metallic), as a function of the relative orientation of the magnetization in the leads. This led to the prediction that the GMR ratio varies with the bias voltage and can change significantly depending on the type of molecule. A similar study investigated the effect of surface states on the magnetoresistance of organic spin valves [165]. In the case of $\mathrm{Ni}$ chains it was found the transport properties depended dramatically on the number of atoms in the chain and on the position of the domain wall inside the junction [55]. It was also studied the possibility of huge magnetoresistances and the effect of oxygen impurities [166]. In the metallocenes calculations it was proved that it is possible to encapsulate such molecules inside carbon nanotubes, which tend to make chains and can lead to GMR ratios when one of the spins is flipped [153]. The electronic and transport properties depend on the type of metallocene and can lead to interesting physical phenomena such as spin spirals [167], charge transfers than can transform semiconducting into metallic nanobubes and wells in the nanotube potential which act as quantum [155].

Similar calculations have also been performed by other groups with other codes (e.g. [56, 57]) and these involve for example the use of cobaltocenes as switches [111] or spin filters [168], non-linear spin current and magnetoresistance in BDT junctions [169], spin injection from nickel contacts to octanethiol [170], inverse magnetoresistance [172], spincurrent rectification [173] and conformation effects on spinpolarized transport [175]. 
(a)
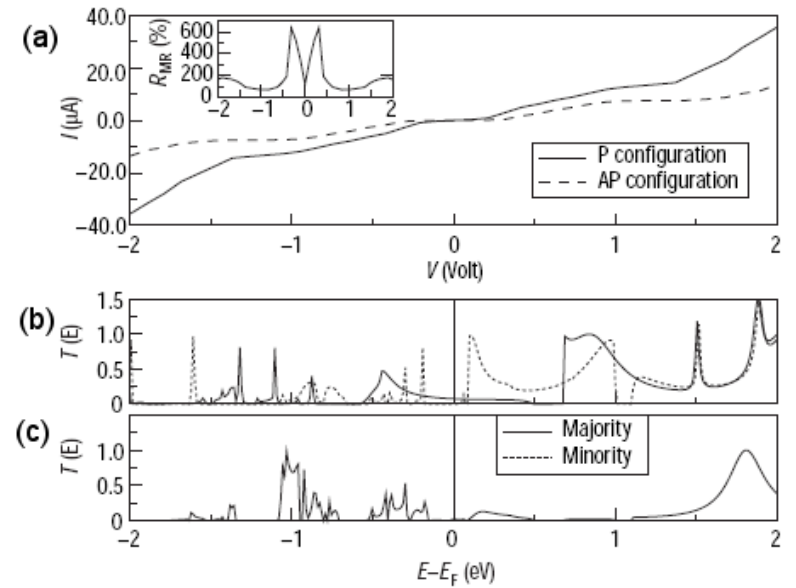

FIG. 19: (Color online) Transport properties of an tricene molecule sandwiched between Ni leads. We plot the current as a function of the bias voltage (a) and the majority and minority transmission coefficients for the parallel and antiparallel configurations (b) and (c). The inset shows the magnetoresistive ratio as a function of bias voltage. Figure taken from [139].

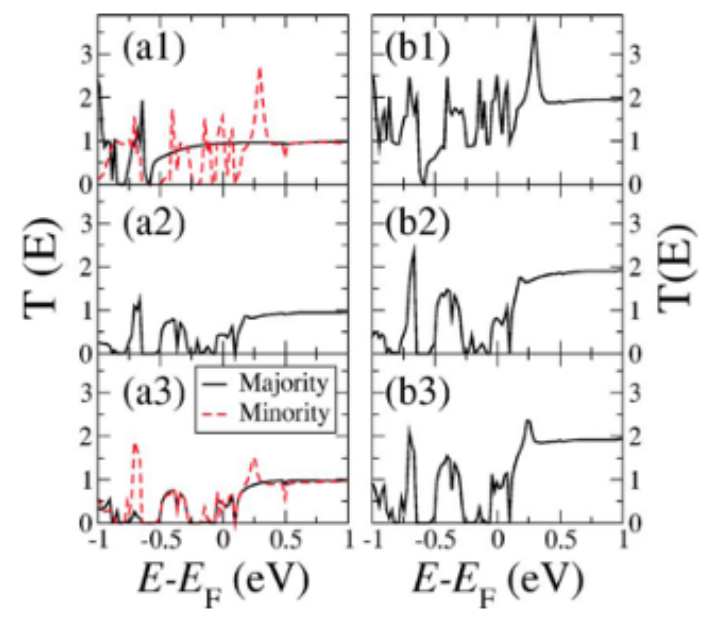

FIG. 20: (Color online) Transmission coefficients for a 4-atoms Ni chain sandwiched between Ni electrodes. The righthand- side panels (a) are for collinear calculations and the left-handside panels (b) are for noncollinear: (1) parallel state, (2) antiparallel with symmetric domain wall, and (3) antiparallel with asymmetric domain wall. Note that in the noncollinear case there is no distinction between majority and minority spins. In panel (a2) majority and minority spins are degenerate. Figure taken from [55].

Apart from the encapsulation of metallocenes, other transport calculations involving nanotubes include spin-dependent transport in iron-doped carbon nanotubes [171], GMR effects in CNT tunnel junctions calculated with a tight-binding model [174] and in CNTs contacted to nickel electrodes calculated from first principles [176]. The problem of dealing with nanotubes between ferromagnetic electrodes comes from

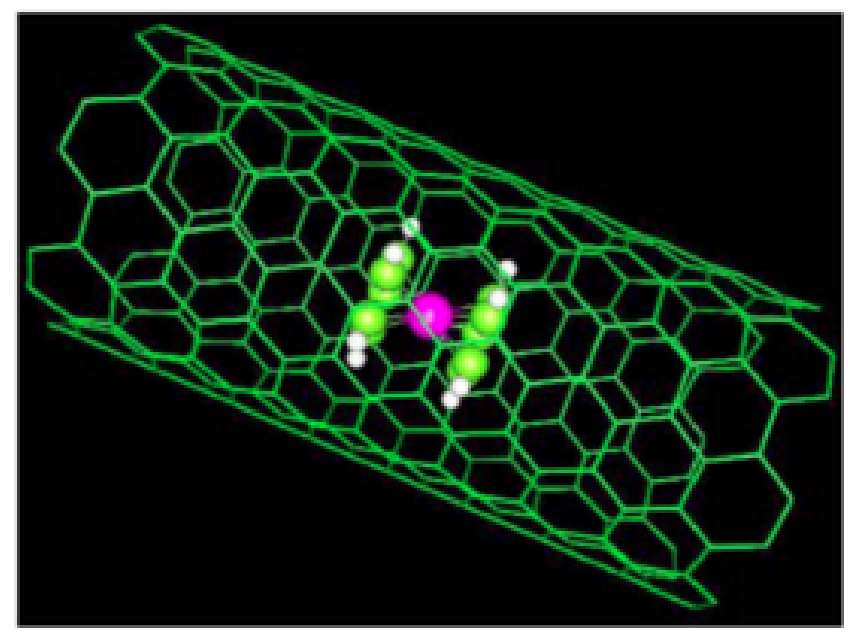

FIG. 21: (Color online) View of a metallocene inside an armchair carbon nanotube. The atoms of the metallocene have been highlighted for clarity. Figure taken from [155].
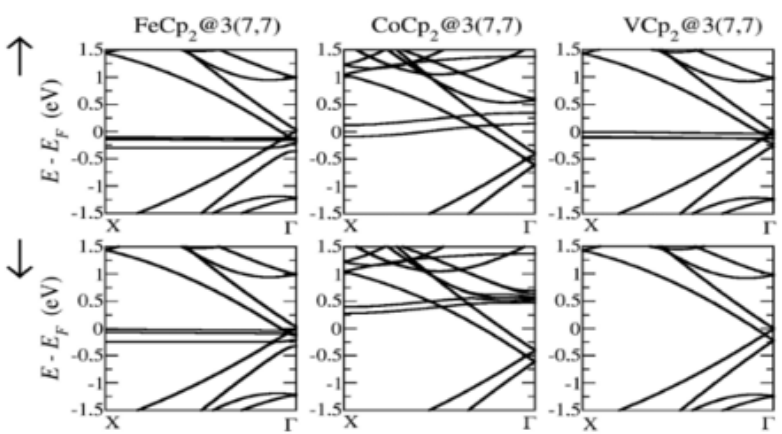

FIG. 22: Band structure of parallel TMCp2@3(7,7) chains, where $\mathrm{TM}=\mathrm{Fe}$, Co, and V. Top (bottom) panels are for spin-up (-down) electrons.

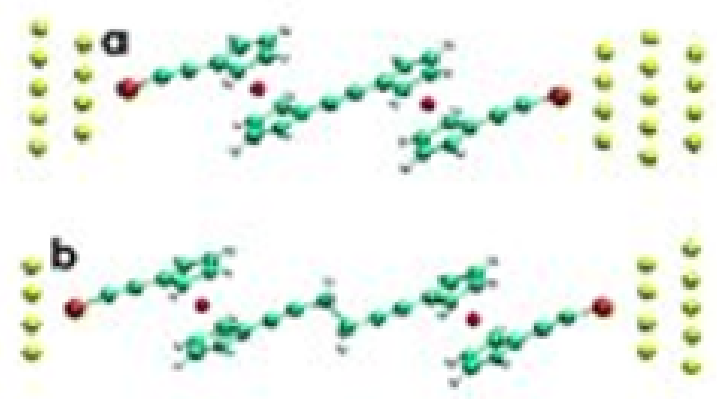

FIG. 23: Example of dicobaltocene switches with different spacers: a pure carbon chain (a) and a carbon chain containing the group $\mathrm{C}_{2} \mathrm{H}_{4}$ (b). Figure taken from [111]. 


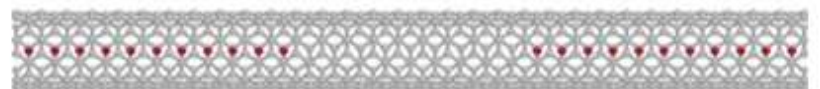

(a)

(b)

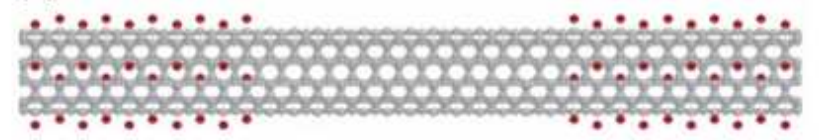

FIG. 24: Examples of carbon nanotubes in contact with Ni electrodes. (a) Ni atoms encapsulated along the axis of the CNT; (b) Ni atoms located on the surface of the CNT. Figure taken from [176].

the fact that such calculations require a lot of atoms and it is not clear how the nanotube will be contacted to the leads. In that sense it is better to use some approximations to include the spin degree of freedom in the leads, such as locate atoms in the nanotube axis or wall [176]. Similar studies have also considered carbon nanotubes on top of magnetic layers and predicted large magnetoresistive ratios [177]. Other spintronic calculations studied also spin transport between carbon nanotubes through organo-metallic benzene-vanadium clusters [178]. Finally, other types of nanotubes made of other types of atoms which also include some magnetic element have also been considered from a theoretical point of view, see for instance [179].

We acknowledge support from the Spanish government and European Commission (project FIS2006-12117 and contract MRTN-CT-2003-504574). JF has profited from conversations with J. I. Martin. G. Rodríguez and L. Fernández-Seivane assisted us with the production of some of the figures.

[1] http://www.forbes.com/lists/

[2] The Economist journal, May 24th 2008.

[3] http://www.intel.com/products/processor/core2xe/specifications.htm[38] X. D. Cui, A. Primak, X. Zarate, J. Tomfohr, O. F. Sankey, A.

[4] International Technology Roadmap for Semiconductors (ITRS), 2005 edition

[5] Building a new semiconductor fabrication plant can cost 5 billion US dollars nowadays.

[6] G. binnig and H. Rohrer, Rev. Mod. Phys. 59, 615 (1986); G. Binnig and H. Rohrer, IBM J. Res. Develop.30, 355 (1986).

[7] R. E. Smalley, Scientific American 285, 76 (2001).

[8] S. Datta, Electronic transport in mesoscopic systems, Cambridge University Press (1995).

[9] N. W. Ashcroft and N. D. Mermin, Solid State Physics, Brooks Cole, 1st edition (1976)

[10] R. Landauer, IBM J. Res. Dev. 1, 223 (1957); M. Büttiker, Phys. Rev. Lett. 57, 1761 (1986).

[11] M. Büttiker, Y. Imry, R. Landauer, and S. Pinhas, Phys. Rev. B 31, 6207 (1985).

[12] N. D. Lang, Phys. Rev. B 36, 8173 (1987).

[13] J. Ferrer, A. Martín-Rodero, and F. Flores, Phys. Rev. B 38, 10113 (1988).

[14] J. Ferrer, Ph. D. Thesis, Universidad Autónoma de Madrid
(1990).

[15] J. C. Cuevas, A. Levy Yeyati and A. Martín-Rodero, Phys. Rev. Lett. 80, 1066 (1998).

[16] A. Aviram and M. A. Ratner, Chem. Phys. Lett. 52, 9071 (1974).

[17] R. A. de Groot, F. M. Mueller, P. G. van Engen and K. H. J. Buschow, Phys. Rev. Lett. 50, 2024 (1983).

[18] I. I. Mazin, Phys. Rev. Lett. 83, 1427 (1999).

[19] P. Bruno, Magnetismus von Festkörpern und grenzflächen, edited by P.H. Dederichs, P. GrÃ $\frac{1}{4}$ nberg, and W. Zinn, Forschungszentrum JÃ $\frac{1}{4}$ lich (1993)

[20] L. Fernández-Seivane and J. Ferrer, Phys. Rev. Lett. 99, 183401 (2007)

[21] T. O. Strandberg, C. M. Canali and A. H. MacDonald, Nature Materials 6, 648 (2007)

[22] K. Tsukagoshi, B. W. Alphenaar, and H. Ago, Nature (London) 401, 572 (1999).

[23] Z. M. Zeng, et al., Phys. Rev. Lett. 97, 106605 (2006).

[24] G. Binasch, P. Grünberg, F. Saurenbach and W. Zinn, Phys. Rev. B 39, 4828 (1989).

[25] M. N. Baibich et al., Phys. Rev. Lett. 61, 2472 (1988).

[26] G. A. Prinz, Science 282, 1660 (1998).

[27] A very illuminating animation of the behavior of both AMR and GMR read heads in a hard drive can be found in http://www.research.ibm.com/research/gmr.html

[28] J. NoguÃ(C)s and I. K. schüller, J. Magn. Magn. Matt. 192, 203 (1999).

[29] J. I. MartÃn, J. NoguÃ(c)s, K. Liu, J. L. Vicent and I. K Schüller, J. Magn. Magn. Matt. 256, 449 (2003).

[30] L. Berger, J. Appl. Phys. 55, 1954 (1984).

[31] L. Berger, Phys. Rev. B 75, 174401 (2007).

[32] S. S. P. Parkin, M. Hayashi and L. Thomas, Science 320, 190 (2008).

[33] C. Joachim, J. K. Gimzewski, R. R. Schlitter, and C. Chavy, Phys. Rev. Lett. 74, 2102 (1995).

[34] S. Datta, W. Tian, S. Hong, R. Reifenberger, J. I. Henderson, and C. P. Kubiak, Phys. Rev. Lett. 79, 2530 (1997).

[35] M. A. Reed, C. Zhou, C. J. Muller, T. P. Burgin, and J. M. Tour, Science 278, 252 (1997). ]

[36] C. J. Muller, J. M. van Ruitenbeek, L. J. de Jongh, Physica C 191, 485 (1992).

[37] W. Haiss, H. van Zalinge, S. J. Higgins, D. Bethell, H. Hobenreich, D. J. Schiffrin, and R. J. Nichols, J. Am. Chem. Soc. 125, 15294 (2003).

L. Moore, T. A. Moore, D. Gust, H. G. Lindsay, and S. M. Lindsay, Science 294, 571 (2001).

[39] B. Xu and N. J. Tao, Science 301, 1221 (2003).

[40] H. Park, A. K. I. Lim, J. Park, A. P. Alivisatos, and P. L. McEuen, Appl. Phys. Lett. 75, 301 (1999).

[41] J. Park, A. N. Pasupathy, J. I. Goldsmith, C. Chang, Y. Yaish, McEuen, and D. C. Ralph, Nature (London) 417, 722 (2002).

[42] W. Liang, M. P. Shores, M. Bockrath, J. R. Long, and H. Park, Nature (London) 417, 725 (2002).

[43] D. J. Wold and C. D. Frisbie, J. Am. Chem. Soc. 122, 2970 (2000).

[44] J. Chen, M. A. Reed, A. M. Rawlett, and J. M. Tour, Science 286, 1550 (1999).

[45] J. G. Kushmerick, D. B. Holt, S. K. Pollack, M. A. Ratner, J. C. Yang, T. L. Schull, J. Naciri, M. H. Moore, and R. Shashidhar, J. Am. Chem. Soc. 124, 10654 (2002).

[46] R. E. Holmlin, R. Haag, M. L. Chabinyc, R. F. Ismagilov, A. J. R. Petta, M. Rinkoski, J. P. Sethna, H. D. Abruñas, P. L. 
E. Cohen, A. Terfort, M. A. Rampi, and G. M. Whitesides, J. Am. Chem. Soc. 123, 5075 (2001).

[47] A. Troisi and M. A. Ratner, Small 2, 172 (2006).

[48] G. Breit and E. Wigner, Phys. Rev. 49, 519 (1936).

[49] U. Fano, Phys. Rev. 124, 1866 (1961).

[50] T. A. Papadopoulos, I. M. Grace and C. J. Lambert, Phys. Rev. B 74, 193306 (2006).

[51] L. V. Keldysh, Sov. Phys. JETP 20, 1018 (1965).

[52] M. Wagner, Phys. Rev. B 44, 6104 (1991)

[53] C. Caroli, R. Rombescot, D. Lederer, P. Nozieres, and D. Saint-James, J. Phys. C 4, 2598 (1971); J. Phys. C 5, 21 (1972).

[54] R. Leeuwen, Int. J. Mod. Phys. B 15, 1969 (2001).

[55] A. R. Rocha, V. M. García-Suárez, S. Bailey, C. Lambert, J. Ferrer and S. Sanvito, Phys. Rev. B 73, 085414 (2006).

[56] J. J. Palacios, A. J. Perez-Jimenez, E. Louis, and J. A. Verges, Phys. Rev. B 64, 115411 (2001).

[57] M. Brandbyge, J.-L. Mozos, P. Ordejón, J. Taylor, and K. Stokbro, Phys. Rev. B 65, 165401 (2002).

[58] I. Rungger and S. Sanvito, http://arxiv.org/abs/0712.1369v1

[59] This is not always possible since in many cases the matrices $\mathcal{G}_{\mathrm{L}, \mathrm{R}}^{0 \mathrm{R}}(E), \Sigma_{L, R}^{R}(E)$ and $\Gamma_{\mathrm{L}, \mathrm{R}}$ are too large.

[60] H. Hohenberg and W. Kohn, Phys. Rev. B 136, B864 (1964).

[61] W. Kohn and L. J. Sham, Phys. Rev. 140, A1133 (1965).

[62] J. P. Perdew and A. Zunger, Phys. Rev. B 23, 5075 (1981)

[63] J. P. Perdew, K. Burke and Ernzerhof Phys. Rev. Lett. 77, 3865 (1996).

[64] A. D. Becke, Phys. Rev. A 38, 3098 (1988); C. Lee, W. Yang and R. G. Parr, Phys. Rev. B 37, 785 (1988)

[65] M. Di Ventra, S. T. Pantelides, and N. D. Lang, Phys. Rev. Lett. 84, 979 (2000).

[66] V. M. García-Suárez, T. Kostyrko, S. Bailey, C. Lambert, and B. R. Bułka, Phys. Stat. Sol. B 244, 2443 (2007).

[67] S. M. Lindsay and M. A. Ratner, Adv. Mater. 19, 23 (2007).

[68] B. C. Stipe, M. A. Rezaei, and W. Ho, Science 280, 1732 (1998).

[69] Z. Ning, J. Chen, S. Hou, J. Zhang, Z. Liang, J. Zhang, and R. Han, Phys. Rev. B 72, 155403 (2005).

[70] A. Filippetti and N. Spalding, Phys. Rev. B 67, 125109 (2003).

[71] C. Toher, S. Sanvito and K. Burke, Phys. Rev. Lett. 95, 146402 (2005).

[72] C. Toher and S. Sanvito, Phys. Rev. B 77, 155402 (2008).

[73] S. H. Ke, H. U. Baranger, and W. T. Yang, J. Chem. Phys. 126, 201102 (2007).

[74] N. Sai, M. Zwolak, G. Vignale, and M. Di Ventra, Phys. Rev. Lett. 94, 186810 (2005).

[75] P. Darancet, A. Ferretti, D. Mayou, and V. Olevano, Phys. Rev. B 75, 075102 (2007).

[76] K. S. Thygesen and A. Rubio, Phys. Rev. B 77, 115333 (2008).

[77] D. S. Fisher and P. A. Lee, Phys. Rev. B 23, R6851 (1981).

[78] Y. Meir and N. S. Wingreen, Phys. Rev. Lett. 68, 2512 (1992).

[79] G. Stefanucci and C.-O. Almbladh, Phys. Rev. B 69, 195318 (2004); G. Stefanucci, S. Kurth, A. Rubio, and E. K. U. Gross, Phys. Rev. B 77, 075339 (2008)

[80] L. Arrachea and M. Moskalets, Phys. Rev. B 74, 245322 (2006).

[81] P. Delaney and J. C. Greer, Phys. Rev. Lett. 93, 036805 (2004).

[82] B. Muralidharan, A. W. Ghosh, S. K. Pati, and S. Datta, IEEE Transactions on Nanotechnology 6, 536 (2007).

[83] J. Gaudioso, L. J. Lauhon, and W. Ho, Phys. Rev. Lett. 85, 1918 (2000).

[84] R. H. M. Smit, Y. Noat, C. Untiedt, N. D. Lang, M. C. van Hemert, and J. M. van Ruitenbeek, Nature (London) 419, 906 (2002).
[85] B. N. J. Persson and A. Baratoff, Phys. Rev. Lett. 59, 339 (1987).

[86] N. Lorente and M. Persson, Phys. Rev. Lett. 85, 2997 (2000).

[87] A. Troisi and M. A. Ratner, Phys. Rev. B 72, 033408 (2005).

[88] N. Sergueev, D. Roubtsov, and H. Guo, Phys. Rev. Lett. 95, 146803 (2005).

[89] T. Frederiksen, Master's Thesis, Technical University of Denmark (2004).

[90] T. Frederiksen, M. Paulsson, M. Brandbyge, and A.-P. Jauho, Phys. Rev. B 75, 205413 (2007).

[91] M. Galperin, A. Nitzan, and M. A. Ratner, Phys. Rev. B 76, 035301 (2007).

[92] J. C. Ellembogen and J. C. Love, Proc. IEEE 88, 386 (2000).

[93] C. Krzeminski, C. Delerue, G. Allan, D. Vuillaume, and R. M. Metzger, Phys. Rev. B 64, 085405 (2001).

[94] P. E. Kornilovitch, A. M. Bratkovsky, and R. Stanley Williams, Phys. Rev. B 66, 165436 (2002).

[95] A. S. Martin, J. R. Sambles, and G. J. Ashwell, Phys. Rev. Lett. 70, 218 (1993).

[96] R. M. Metzger, B. Chen, U. Höpfner, M. V. Lakshmikantham, D. Vuillaume, T. Kawai, X. Wu, H. Tachibana, T. V. Hughes, H. Sakurai, J. W. Baldwin, C. Hosch, M. P. Cava, L. Brehmer, and G. J. Ashwell, J. Am. Chem. Soc. 119, 10455 (1997).

[97] C. Zhou, M. R. Deshpande, M. A. Reed, L. Jones II, and J. M. Tour, Appl. Phys. Lett. 71, 611 (1997).

[98] Z. Yao, H. W. Ch. Postma, L. Balents, and C. Dekker, Nature (London) 402, 273 (1999).

[99] M. Galperin and A. Nitzan, Phys. Rev. Lett. 95, 206802 (2005).

[100] E. Flaxer, O. Sneh, and O. Cheshnovsky, Science 262, 2012 (1993).

[101] X. H. Qiu, G. V. Nazin, and W. Ho, Science 299, 542 (2003).

[102] M. Switkes, C. M. Marcus, K. Campman, and A. C. Gossard, Science 283, 1905 (1999).

[103] J. Lehmann, S. Camalet, S. Kohler, and P. Hänggi, Chem. Phys. Lett. 368, 282 (2002).

[104] A. Scott and J. R. Sambles, Nanotechnology 7, 401 (1996).

[105] B. L. Feringa, Molecular Switches, Wiley-VCH, Weinheim (2001).

[106] Z. J. Donhauser, B. A. Mantooth, K. F. Kelly, L. A. Bumm, J. D. Monnell, J. J. Stapleton, D. W. Price Jr., A. M. Rawlett, D. L. Allara, J. M. Tour, and P. S. Weiss, Science 292, 2303 (2001).

[107] P. G. Piva, G. A. DiLabio, J. L. Pitters, J. Zikovsky, M. Rezeq, S. Dogel, W. A. Hofer, and R. A. Wolkow, Nature (London) 435, 658 (2005).

[108] C. Joachim and J. K. Gimzewski, Chem. Phys. Lett. 265, 353 (1997).

[109] E. G. Emberly and G. Kirczenow, Phys. Rev. Lett. 91, 188301 (2003).

[110] Y.-H. Kim, S. S. Jang, Y. H. Jang, and W. A. Goddard III, Phys. Rev. Lett. 94, 156801 (2005).

[111] R. Liu, S.-H. Ke, H. U. Baranger, and W. Yang, Nano Lett. 5, 1959 (2005).

[112] C. P. Collier, E. W. Wong, M. Belohradský, F. M. Raymo, J. F. Stoddart, P. J. Kuekes, R. S. Williams, and J. R. Heath, Science 285, 391 (1999).

[113] S. J. Tans, A. R. M. Verschueren, and C. Dekker, Nature (London) 393, 49 (1998).

[114] J. H. Schön, H. Meng, and Z. Bao, Science 294, 2138 (2001).

[115] S. Kubatkin, A. Danilov, M. Hjort, J. Cornill, J.-L. Brédas, N. Stuhr-Hansen, P. Hedegàrd, and T. Bjørnholm, Nature (London) 425, 698 (2003).

[116] R. L. Carroll and C. B. Gorman, Angew: Chem. Int. Ed. 41, 
4379 (2002).

[117] Z. Crljen and G. Baranović, Phys. Rev. Lett. 98, 116801 (2007).

[118] L. Esaki, Phys. Rev. 109, 603 (1958).

[119] S. M. Sze, Physics of Semiconductor Devices (Wiley, New York, 1981), p. 190.

[120] H. Eisele and G. I. Haddad, in Modern Semiconductor Device Physics, edited by S. M. Sze (Wiley, New York, 1998), p. 343.

[121] T. Rakshit, G.-C. Liang, A. W. Ghosh, M. C. Hersam, and S. Datta, Phys. Rev. B 72, 125305 (2005).

[122] I.-W. Lyo and Ph. Avouris, Science 245, 1369 (1989).

[123] H. Dalgleish and G. Kirczenow, Phys. Rev. B 73, 245431 (2006).

[124] L. Chen, Z. Hu, A. Zhao, B. Wang, Y. Luo, J. Yang, and J. G. Hou, Phys. Rev. Lett. 99, 146803 (2007).

[125] F. Chen, X. Li, H. Hihath, Z. Huang, and N. Tao, J. Am. Chem. Soc. 128, 15874 (2006).

[126] D. P. Long, J. L. Lazorcik, B. A. Mantooth, M. H. Moore, M. A. Ratner, A. Troisi, Y. Yao, J. W. Ciszek, J. M. Tour, and R. Shashidhar, Nat. Mater 5, 901 (2006).

[127] K. Schwab, E. A. Henriksen, J. M. Worlock, and M. L. Roukes, Nature (London) 404, 974 (2000).

[128] M. Galperin, A. Nitzan, and M. A. Ratner, Phys. Rev. B 75, 155312 (2007).

[129] J. C. Poler, R. M. Zimmermann, and E. C. Cox, Langmuir 11, 2689 (1995).

[130] M. Paulsson and S. Datta, Phys. Rev. B 67, 241403R (2003).

[131] V. Dediu, M. Murgia, F. C. Matacotta, C. Taliani, and S. Barbanera, Solid State Commun. 122, 181 (2002).

[132] M. Ouyang and D. D. Awschalom, Science 301, 1074 (2003).

[133] J. R. Petta, S. K. Slater, and D. C. Ralph, Phys. Rev. Lett. 93, 136601 (2004).

[134] Z. H. Xiong, D. Wu, Z. Valy Vardeny, and J. Shi, Nature (London) 427, 821 (2004).

[135] E. G. Emberly and G. Kirczenow, Chem. Phys. 281, 311 (2002).

[136] R. Pati, M. Mailman, L. Senapati, P. M. Ajayan, S. D. Mahanti, and S. K. Nayak, Phys. Rev. B 68, 014412 (2003).

[137] R. Pati, L. Senapati, P. M. Ajayan, and S. K. Nayak, Phys. Rev. B 68, 100407R (2003).

[138] Y. Wei, Y. Xu, J. Wang, and H. Guo, Phys. Rev. B 70, 193406 (2004).

[139] A. R. Rocha, V. M. García-Suárez, S. W. Bailey, C. J. Lambert, J. Ferrer, and S. Sanvito, Nature Materials 4, 335 (2005).

[140] S. Sanvito and A. R. Rocha, J. Comput. Theor. Nanosci. 3, 624 (2006).

[141] L. Bogani and W. Wernsdorfer, Nature Materials 7, 179 (2008).

[142] D. Gatteschi, R. Sessoli, and J. Villain, Molecular Nanomagnets (Oxford Univ. Press, New York, 2007).

[143] J. R. Friedman, M. P. Sarachik, J. Tejada, and R. Ziolo, Phys. Rev. Lett. 76, 3830 (1996).

[144] L. Thomas, F. Lionti, R. Ballou, D. Gatteschi, R. Sessoli, and B. Barbara, Nature (London) 383, 145 (1996).

[145] W. Wernsdorfer and R. Sessoli, Science 284, 133 (1999).

[146] M.-H. Jo, J. E. Grose, K. Baheti, M. M. Deshmukh, J. J. Sokol, E. M. Rumberger, D. N. Hendrikson, J. R. Long, H. Park, and D. C. Ralph, Nano Letters 6, 2014 (2006).

[147] C. Romeike, M. R. Wegewijs, W. Hofstetter, and H. Schoeller, Phys. Rev. Lett. 97, 196601 (2006).

[148] C. Romeike, M. R. Wegewijs, W. Hofstetter, and H. Schoeller, Phys. Rev. Lett. 97, 206601 (2006).

[149] A. V. Postnikov, J. Kortus, and M. R. Pederson, Phys. Stat.
Solidi B 243, 2533 (2006).

[150] M. N. Leuenberger and D. Loss, Nature (London) 410, 789 (2001).

[151] A. K. Ekert and R. Jozsa, Rev. Mod. Phys. 68, 733 (1996).

[152] M. Habgood, J. H. Jefferson, A. Ramsăk, D. G. Pettifor, and G. A. D. Briggs, Phys. Rev. B 77, 075337 (2008).

[153] V. M. García-Suárez, J. Ferrer, and C. J. Lambert, Phys. Rev. Lett. 96, 106804 (2006).

[154] L. J. Li, A. N. Khlobystov, J. G. Wiltshire, G. A. D. Briggs, and R. J. Nicholas, Nature Materials 4, 481 (2005).

[155] V. M. García-Suárez, J. Ferrer, and C. J. Lambert, Phys. Rev. B 74, 205421 (2006).

[156] P. Bruno, Phys. Rev. Lett. 83, 2425 (1999).

[157] V. M. García-Suárez and J. Ferrer, unpublished.

[158] V. M. García-Suárez, D. Manrique, C. J. Lambert and J. Ferrer, submitted.

[159] L. Fernández-Seivane, M. A. Oliveira, S. Sanvito and J. Ferrer, J. Phys.: Condens. Matter 18, 7999 (2006).

[160] S. Sanvito, C. J. Lambert, J. H. Jefferson, and A. M. Bratkovsky, Phys. Rev. B 59, 11936 (1999).

[161] M. B. Nardelli, Phys. Rev. B 60, 7828 (1999).

[162] L. Fernández-Seivane, V. M. García-Suárez and J. Ferrer, Phys. Rev. B 75, 075415 (2007).

[163] J. M. Soler, E. Artacho, J. D. Gale, A. García, J. Junquera, P. Ordejón, and D. Sánchez-Portal, J. Phys.: Condens. Matter 14, 2745 (2002).

[164] A. Smogunov, A. Dal Corso, and E. Tosatti, arXiv:0804.3340 (2008).

[165] A. R. Rocha and S. Sanvito, Journal of Applied Physics 101, 09B102 (2007).

[166] A. R. Rocha, T. Archer, and S. Sanvito, Phys. Rev. B 76, 054435 (2007).

[167] V. M. García-Suárez, C. M. Newman, C. J. Lambert, J. M. Pruneda, and J. Ferrer, J. Phys.: Condens. Matter 16, 5453 (2004).

[168] R. Liu, S.-H. Ke, W. Yang, and H. U. Baranger, J. Chem. Phys. 127, 141104 (2007).

[169] D. Waldron, P. Haney, B. Larade, A. MacDonald, and H. Guo, Phys. Rev. Lett. 96, 166804 (2006).

[170] Z. Ning, Y. Zhu, J. Wang, and H. Guo, Phys. Rev. Lett. 100, 056803 (2008).

[171] B. Wang, Y. Zhu, W. Ren, J. Wang, and H. Guo, Phys. Rev. B 75, 235415 (2007).

[172] H. Dalgleish and G. Kirczenow, Phys. Rev. B 72, 184407 (2005).

[173] H. Dalgleish and G. Kirczenow, Phys. Rev. B 73, 235436 (2006).

[174] H. Mehrez, J. Taylor, H. Guo, J. Wang, and C. Roland, Phys. Rev. Lett. 84, 2682 (2000).

[175] L. Senapati, R. Pati, and S. C. Erwin, Phys. Rev. B 76, 024438 (2007).

[176] S. Athanasopoulos, S. W. Bailey, J. Ferrer, V. M. GarcíaSuárez, C. J. Lambert, A. R. Rocha, and S. Sanvito, J. Phys.: Condens. Matter 19, 042201 (2007).

[177] L. E. Hueso, J. M. Pruneda, V. Ferrari, G. Burnell, J. P. ValdesHerrera, B. D. Simons, P. B. Littlewood, E. Artacho, A. Fert, and N. D. Mathur, Nature (London) 445, 410 (2007).

[178] M. Koleini, M. Paulsson, and M. Brandbyge, Phys. Rev. Lett. 98, 197202 (2007)

[179] B. Sanyal, O. Eriksson, and P. I. Arvidsson, Phys. Rev. B 77, 155407 (2008). 